\title{
Positive solutions for a system of semipositone coupled fractional boundary value problems
}

\author{
Johnny Henderson ${ }^{1}$ and Rodica Luca ${ }^{2 *}$
}

${ }^{\text {"Correspondence: }}$

rluca@math.tuiasi.ro

${ }^{2}$ Department of Mathematics,

Gh. Asachi Technical University, lasi,

700506, Romania

Full list of author information is

available at the end of the article

\section{Springer}

\begin{abstract}
We study the existence of positive solutions for a system of nonlinear Riemann-Liouville fractional differential equations with sign-changing nonlinearities, subject to coupled integral boundary conditions.
\end{abstract}

MSC: $34 \mathrm{A08} ; 45 \mathrm{G} 15$

Keywords: Riemann-Liouville fractional differential equations; coupled integral boundary conditions; positive solutions; sign-changing nonlinearities

\section{Introduction}

Fractional differential equations describe many phenomena in various fields of engineering and scientific disciplines such as physics, biophysics, chemistry, biology, economics, control theory, signal and image processing, aerodynamics, viscoelasticity, electromagnetics, and so on (see [1-6]). Integral boundary conditions arise in thermal conduction problems, semiconductor problems and hydrodynamic problems.

We consider the system of nonlinear fractional differential equations

$$
\text { (S) } \begin{cases}D_{0+}^{\alpha} u(t)+\lambda f(t, u(t), v(t))=0, & t \in(0,1), n-1<\alpha \leq n, \\ D_{0+}^{\beta} v(t)+\mu g(t, u(t), v(t))=0, & t \in(0,1), m-1<\beta \leq m,\end{cases}
$$

with the coupled integral boundary conditions

$$
(\mathrm{BC}) \begin{cases}u(0)=u^{\prime}(0)=\cdots=u^{(n-2)}(0)=0, & u(1)=\int_{0}^{1} v(s) d H(s), \\ v(0)=v^{\prime}(0)=\cdots=v^{(m-2)}(0)=0, & v(1)=\int_{0}^{1} u(s) d K(s),\end{cases}
$$

where $n, m \in \mathbb{N}, n, m \geq 3, D_{0_{+}}^{\alpha}$, and $D_{0_{+}}^{\beta}$ denote the Riemann-Liouville derivatives of orders $\alpha$ and $\beta$, respectively, the integrals from (BC) are Riemann-Stieltjes integrals, and $f$, $g$ are sign-changing continuous functions (that is, we have a so-called system of semipositone boundary value problems). These functions may be nonsingular or singular at $t=0$ and/or $t=1$. The boundary conditions above include multi-point and integral boundary conditions and sum of these in a single framework.

We present intervals for parameters $\lambda$ and $\mu$ such that the above problem (S)-(BC) has at least one positive solution. By a positive solution of problem (S)-(BC) we mean a pair

(c) 2016 Henderson and Luca. This article is distributed under the terms of the Creative Commons Attribution 4.0 International License (http://creativecommons.org/licenses/by/4.0/), which permits unrestricted use, distribution, and reproduction in any medium, provided you give appropriate credit to the original author(s) and the source, provide a link to the Creative Commons license, and indicate if changes were made. 
of functions $(u, v) \in C([0,1]) \times C([0,1])$ satisfying (S) and (BC) with $u(t) \geq 0, v(t) \geq 0$ for all $t \in[0,1]$ and $u(t)>0, v(t)>0$ for all $t \in(0,1)$. In the case when $f$ and $g$ are nonnegative, problem $(\mathrm{S})-(\mathrm{BC})$ has been investigated in [7] by using the Guo-Krasnosel'skii fixed point theorem, and in [8] where $\lambda=\mu=1$ and $f(t, u, v)$ and $g(t, u, v)$ are replaced by $\tilde{f}(t, v)$ and $\tilde{g}(t, u)$, respectively (denoted by $(\widetilde{\mathrm{S}})$ ). In [8], the authors study two cases: $f$ and $g$ are nonsingular and singular functions and they used some theorems from the fixed point index theory and the Guo-Krasnosel'skii fixed point theorem. The systems (S) and $(\widetilde{S})$ with uncoupled boundary conditions

$$
(\widetilde{\mathrm{BC}}) \begin{cases}u(0)=u^{\prime}(0)=\cdots=u^{(n-2)}(0)=0, & u(1)=\int_{0}^{1} u(s) d H(s), \\ v(0)=v^{\prime}(0)=\cdots=v^{(m-2)}(0)=0, & v(1)=\int_{0}^{1} v(s) d K(s),\end{cases}
$$

were investigated in [9] (problem (S)-( $\widetilde{\mathrm{BC}})$ with $f, g$ nonnegative), in [10] (problem $(\widetilde{\mathrm{S}})$ $(\widetilde{\mathrm{BC}})$ with $f, g$ nonnegative, singular or not), and in [11] (problem (S)-( $\widetilde{\mathrm{BC}})$ with $f, g$ signchanging functions). We also mention paper [12], where the authors studied the existence and multiplicity of positive solutions for system (S) with $\alpha=\beta, \lambda=\mu$, and the boundary conditions $u^{(i)}(0)=v^{(i)}(0)=0, i=0, \ldots, n-2, u(1)=a v(\xi), v(1)=b u(\eta), \xi, \eta \in(0,1)$, with $\xi, \eta \in(0,1), 0<a b \xi \eta<1$, and $f, g$ are sign-changing nonsingular or singular functions.

The paper is organized as follows. Section 2 contains some preliminaries and lemmas. The main results are presented in Section 3, and finally in Section 4 some examples are given to support the new results.

\section{Auxiliary results}

We present here the definitions of Riemann-Liouville fractional integral and RiemannLiouville fractional derivative and then some auxiliary results that will be used to prove our main results.

Definition 2.1 The (left-sided) fractional integral of order $\alpha>0$ of a function $f:(0, \infty) \rightarrow$ $\mathbb{R}$ is given by

$$
\left(I_{0+}^{\alpha} f\right)(t)=\frac{1}{\Gamma(\alpha)} \int_{0}^{t}(t-s)^{\alpha-1} f(s) d s, \quad t>0,
$$

provided the right-hand side is pointwise defined on $(0, \infty)$, where $\Gamma(\alpha)$ is the Euler gamma function defined by $\Gamma(\alpha)=\int_{0}^{\infty} t^{\alpha-1} e^{-t} d t, \alpha>0$.

Definition 2.2 The Riemann-Liouville fractional derivative of order $\alpha \geq 0$ for a function $f:(0, \infty) \rightarrow \mathbb{R}$ is given by

$$
\left(D_{0+}^{\alpha} f\right)(t)=\left(\frac{d}{d t}\right)^{n}\left(I_{0+}^{n-\alpha} f\right)(t)=\frac{1}{\Gamma(n-\alpha)}\left(\frac{d}{d t}\right)^{n} \int_{0}^{t} \frac{f(s)}{(t-s)^{\alpha-n+1}} d s, \quad t>0
$$

where $n=\lfloor\alpha\rfloor+1$, provided that the right-hand side is pointwise defined on $(0, \infty)$.

The notation $\lfloor\alpha\rfloor$ stands for the largest integer not greater than $\alpha$. If $\alpha=m \in \mathbb{N}$ then $D_{0+}^{m} f(t)=f^{(m)}(t)$ for $t>0$, and if $\alpha=0$ then $D_{0+}^{0} f(t)=f(t)$ for $t>0$. 
We consider now the fractional differential system

$$
\left\{\begin{array}{l}
D_{0+}^{\alpha} u(t)+\tilde{x}(t)=0, \quad t \in(0,1), n-1<\alpha \leq n, \\
D_{0+}^{\beta} v(t)+\tilde{y}(t)=0, \quad t \in(0,1), m-1<\beta \leq m,
\end{array}\right.
$$

with the coupled integral boundary conditions

$$
\begin{cases}u(0)=u^{\prime}(0)=\cdots=u^{(n-2)}(0)=0, & u(1)=\int_{0}^{1} v(s) d H(s), \\ v(0)=v^{\prime}(0)=\cdots=v^{(m-2)}(0)=0, & v(1)=\int_{0}^{1} u(s) d K(s),\end{cases}
$$

where $n, m \in \mathbb{N}, n, m \geq 3$, and $H, K:[0,1] \rightarrow \mathbb{R}$ are functions of bounded variation.

Lemma 2.1 ([7]) If $H, K:[0,1] \rightarrow \mathbb{R}$ are functions of bounded variations, $\Delta=1$ $\left(\int_{0}^{1} \tau^{\alpha-1} d K(\tau)\right)\left(\int_{0}^{1} \tau^{\beta-1} d H(\tau)\right) \neq 0$ and $\tilde{x}, \tilde{y} \in C(0,1) \cap L^{1}(0,1)$, then the pair of functions $(u, v) \in C([0,1]) \times C([0,1])$ given by

$$
\begin{cases}u(t)=\int_{0}^{1} G_{1}(t, s) \tilde{x}(s) d s+\int_{0}^{1} G_{2}(t, s) \tilde{y}(s) d s, & t \in[0,1] \\ v(t)=\int_{0}^{1} G_{3}(t, s) \tilde{y}(s) d s+\int_{0}^{1} G_{4}(t, s) \tilde{x}(s) d s, & t \in[0,1]\end{cases}
$$

where

$$
\left\{\begin{array}{l}
G_{1}(t, s)=g_{1}(t, s)+\frac{t^{\alpha-1}}{\Delta}\left(\int_{0}^{1} \tau^{\beta-1} d H(\tau)\right)\left(\int_{0}^{1} g_{1}(\tau, s) d K(\tau)\right), \\
G_{2}(t, s)=\frac{t^{\alpha-1}}{\Delta} \int_{0}^{1} g_{2}(\tau, s) d H(\tau), \\
G_{3}(t, s)=g_{2}(t, s)+\frac{t^{\beta-1}}{\Delta}\left(\int_{0}^{1} \tau^{\alpha-1} d K(\tau)\right)\left(\int_{0}^{1} g_{2}(\tau, s) d H(\tau)\right), \\
G_{4}(t, s)=\frac{t^{\beta-1}}{\Delta} \int_{0}^{1} g_{1}(\tau, s) d K(\tau), \quad \forall t, s \in[0,1]
\end{array}\right.
$$

and

$$
\left\{\begin{array}{l}
g_{1}(t, s)=\frac{1}{\Gamma(\alpha)} \begin{cases}t^{\alpha-1}(1-s)^{\alpha-1}-(t-s)^{\alpha-1}, & 0 \leq s \leq t \leq 1, \\
t^{\alpha-1}(1-s)^{\alpha-1}, & 0 \leq t \leq s \leq 1,\end{cases} \\
g_{2}(t, s)=\frac{1}{\Gamma(\beta)} \begin{cases}t^{\beta-1}(1-s)^{\beta-1}-(t-s)^{\beta-1}, & 0 \leq s \leq t \leq 1, \\
t^{\beta-1}(1-s)^{\beta-1}, & 0 \leq t \leq s \leq 1,\end{cases}
\end{array}\right.
$$

is solution of problem (1)-(2).

Lemma 2.2 The functions $g_{1}, g_{2}$ given by (5) have the properties:

(a) $g_{1}, g_{2}:[0,1] \times[0,1] \rightarrow \mathbb{R}_{+}$are continuous functions, and $g_{1}(t, s)>0, g_{2}(t, s)>0$ for all $(t, s) \in(0,1) \times(0,1)$.

(b) $g_{1}(t, s) \leq h_{1}(s), g_{2}(t, s) \leq h_{2}(s)$ for all $(t, s) \in[0,1] \times[0,1]$, where $h_{1}(s)=\frac{s(1-s)^{\alpha-1}}{\Gamma(\alpha-1)}$ and $h_{2}(s)=\frac{s(1-s)^{\beta-1}}{\Gamma(\beta-1)}$ for all $s \in[0,1]$.

(c) $g_{1}(t, s) \geq k_{1}(t) h_{1}(s), g_{2}(t, s) \geq k_{2}(t) h_{2}(s)$ for all $(t, s) \in[0,1] \times[0,1]$, where

$$
\begin{aligned}
& k_{1}(t)=\min \left\{\frac{(1-t) t^{\alpha-2}}{\alpha-1}, \frac{t^{\alpha-1}}{\alpha-1}\right\}= \begin{cases}\frac{t^{\alpha-1}}{\alpha-1}, & 0 \leq t \leq \frac{1}{2}, \\
\frac{(1-t) t^{\alpha-2}}{\alpha-1}, & \frac{1}{2} \leq t \leq 1,\end{cases} \\
& k_{2}(t)=\min \left\{\frac{(1-t) t^{\beta-2}}{\beta-1}, \frac{t^{\beta-1}}{\beta-1}\right\}= \begin{cases}\frac{t^{\beta-1}}{\beta-1}, & 0 \leq t \leq \frac{1}{2}, \\
\frac{(1-t) t^{\beta-2}}{\beta-1}, & \frac{1}{2} \leq t \leq 1\end{cases}
\end{aligned}
$$


(d) For any $(t, s) \in[0,1] \times[0,1]$, we have

$$
g_{1}(t, s) \leq \frac{(1-t) t^{\alpha-1}}{\Gamma(\alpha-1)} \leq \frac{t^{\alpha-1}}{\Gamma(\alpha-1)}, \quad g_{2}(t, s) \leq \frac{(1-t) t^{\beta-1}}{\Gamma(\beta-1)} \leq \frac{t^{\beta-1}}{\Gamma(\beta-1)} .
$$

For the proof of Lemma 2.2(a) and (b) see [13], for the proof of Lemma 2.2(c) see [11], and the proof of Lemma 2.2(d) is based on the relations $g_{1}(t, s)=g_{1}(1-s, 1-t), g_{2}(t, s)=$ $g_{2}(1-s, 1-t)$, and relations (b) above.

Lemma 2.3 ([7]) If $H, K:[0,1] \rightarrow \mathbb{R}$ are nondecreasing functions, and $\Delta>0$, then $G_{i}$, $i=1, \ldots, 4$ given by (4) are continuous functions on $[0,1] \times[0,1]$ and satisfy $G_{i}(t, s) \geq 0$ for all $(t, s) \in[0,1] \times[0,1], i=1, \ldots, 4$. Moreover, if $\tilde{x}, \tilde{y} \in C(0,1) \cap L^{1}(0,1)$ satisfy $\tilde{x}(t) \geq 0$, $\tilde{y}(t) \geq 0$ for all $t \in(0,1)$, then the solution $(u, v)$ of problem (1)-(2) given by (3) satisfies $u(t) \geq 0, v(t) \geq 0$ for all $t \in[0,1]$.

Lemma 2.4 Assume that $H, K:[0,1] \rightarrow \mathbb{R}$ are nondecreasing functions, $\Delta>0, \int_{0}^{1} \tau^{\alpha-1}(1-$ $\tau) d K(\tau)>0, \int_{0}^{1} \tau^{\beta-1}(1-\tau) d H(\tau)>0$. Then the functions $G_{i}, i=1, \ldots, 4$ satisfy the inequalities:

$\left(\mathrm{a}_{1}\right) G_{1}(t, s) \leq \sigma_{1} h_{1}(s), \forall(t, s) \in[0,1] \times[0,1]$, where

$$
\sigma_{1}=1+\frac{1}{\Delta}(K(1)-K(0)) \int_{0}^{1} \tau^{\beta-1} d H(\tau)>0 .
$$

$\left(\mathrm{a}_{2}\right) G_{1}(t, s) \leq \delta_{1} t^{\alpha-1}, \forall(t, s) \in[0,1] \times[0,1]$, where

$$
\delta_{1}=\frac{1}{\Gamma(\alpha-1)}\left[1+\frac{1}{\Delta}\left(\int_{0}^{1} \tau^{\beta-1} d H(\tau)\right)\left(\int_{0}^{1}(1-\tau) \tau^{\alpha-1} d K(\tau)\right)\right]>0 .
$$

$\left(a_{3}\right) G_{1}(t, s) \geq \varrho_{1} t^{\alpha-1} h_{1}(s),(t, s) \in[0,1] \times[0,1]$, where

$$
\varrho_{1}=\frac{1}{\Delta}\left(\int_{0}^{1} \tau^{\beta-1} d H(\tau)\right)\left(\int_{0}^{1} k_{1}(\tau) d K(\tau)\right)>0 .
$$

$\left(\mathrm{b}_{1}\right) G_{2}(t, s) \leq \sigma_{2} h_{2}(s), \forall(t, s) \in[0,1] \times[0,1]$, where $\sigma_{2}=\frac{1}{\Delta}(H(1)-H(0))>0$.

$\left(\mathrm{b}_{2}\right) G_{2}(t, s) \leq \delta_{2} t^{\alpha-1}, \forall(t, s) \in[0,1] \times[0,1]$, where $\delta_{2}=\frac{1}{\Delta \Gamma(\beta-1)} \int_{0}^{1}(1-\tau) \tau^{\beta-1} d H(\tau)>0$.

$\left(\mathrm{b}_{3}\right) G_{2}(t, s) \geq \varrho_{2} t^{\alpha-1} h_{2}(s), \forall(t, s) \in[0,1] \times[0,1]$, where $\varrho_{2}=\frac{1}{\Delta} \int_{0}^{1} k_{2}(\tau) d H(\tau)>0$.

$\left(\mathrm{c}_{1}\right) G_{3}(t, s) \leq \sigma_{3} h_{2}(s), \forall(t, s) \in[0,1] \times[0,1]$, where

$$
\sigma_{3}=1+\frac{1}{\Delta}(H(1)-H(0)) \int_{0}^{1} \tau^{\alpha-1} d K(\tau)>0 .
$$

$\left(c_{2}\right) \quad G_{3}(t, s) \leq \delta_{3} t^{\beta-1}, \forall(t, s) \in[0,1] \times[0,1]$, where

$$
\delta_{3}=\frac{1}{\Gamma(\beta-1)}\left[1+\frac{1}{\Delta}\left(\int_{0}^{1} \tau^{\alpha-1} d K(\tau)\right)\left(\int_{0}^{1}(1-\tau) \tau^{\beta-1} d H(\tau)\right)\right]>0 .
$$

$\left(c_{3}\right) G_{3}(t, s) \geq \varrho_{3} t^{\beta-1} h_{2}(s), \forall(t, s) \in[0,1] \times[0,1]$, where

$$
\varrho_{3}=\frac{1}{\Delta}\left(\int_{0}^{1} \tau^{\alpha-1} d K(\tau)\right)\left(\int_{0}^{1} k_{2}(\tau) d H(\tau)\right)>0 .
$$


$\left(\mathrm{d}_{1}\right) G_{4}(t, s) \leq \sigma_{4} h_{1}(s), \forall(t, s) \in[0,1] \times[0,1]$, where $\sigma_{4}=\frac{1}{\Delta}(K(1)-K(0))>0$.

$\left(\mathrm{d}_{2}\right) G_{4}(t, s) \leq \delta_{4} t^{\beta-1}, \forall(t, s) \in[0,1] \times[0,1]$, where $\delta_{4}=\frac{1}{\Delta \Gamma(\alpha-1)} \int_{0}^{1}(1-\tau) \tau^{\alpha-1} d K(\tau)>0$.

$\left(\mathrm{d}_{3}\right) G_{4}(t, s) \geq \varrho_{4} t^{\beta-1} h_{1}(s), \forall(t, s) \in[0,1] \times[0,1]$, where $\varrho_{4}=\frac{1}{\Delta} \int_{0}^{1} k_{1}(\tau) d K(\tau)>0$.

Proof From the assumptions of this lemma, we obtain

$$
\begin{aligned}
& \int_{0}^{1} \tau^{\alpha-1} d K(\tau) \geq \int_{0}^{1} \tau^{\alpha-1}(1-\tau) d K(\tau)>0, \\
& \int_{0}^{1}(1-\tau) \tau^{\alpha-2} d K(\tau) \geq \int_{0}^{1}(1-\tau) \tau^{\alpha-1} d K(\tau)>0, \\
& \int_{0}^{1} k_{1}(\tau) d K(\tau) \geq \frac{1}{\alpha-1} \int_{0}^{1} \tau^{\alpha-1}(1-\tau) d K(\tau)>0, \\
& \int_{0}^{1} \tau^{\beta-1} d H(\tau) \geq \int_{0}^{1} \tau^{\beta-1}(1-\tau) d H(\tau)>0, \\
& \int_{0}^{1}(1-\tau) \tau^{\beta-2} d H(\tau) \geq \int_{0}^{1}(1-\tau) \tau^{\beta-1} d H(\tau)>0, \\
& \int_{0}^{1} k_{2}(\tau) d H(\tau) \geq \frac{1}{\beta-1} \int_{0}^{1} \tau^{\beta-1}(1-\tau) d H(\tau)>0, \\
& K(1)-K(0)=\int_{0}^{1} d K(\tau) \geq \int_{0}^{1} \tau^{\alpha-1}(1-\tau) d K(\tau)>0, \\
& H(1)-H(0)=\int_{0}^{1} d H(\tau) \geq \int_{0}^{1} \tau^{\beta-1}(1-\tau) d H(\tau)>0 .
\end{aligned}
$$

By using Lemma 2.2, we deduce, for all $(t, s) \in[0,1] \times[0,1]$ :

$\left(\mathrm{a}_{1}\right)$

$$
\begin{aligned}
G_{1}(t, s) & =g_{1}(t, s)+\frac{t^{\alpha-1}}{\Delta}\left(\int_{0}^{1} \tau^{\beta-1} d H(\tau)\right)\left(\int_{0}^{1} g_{1}(\tau, s) d K(\tau)\right) \\
& \leq h_{1}(s)+\frac{1}{\Delta}\left(\int_{0}^{1} \tau^{\beta-1} d H(\tau)\right)\left(\int_{0}^{1} h_{1}(s) d K(\tau)\right) \\
& =h_{1}(s)\left[1+\frac{1}{\Delta}(K(1)-K(0)) \int_{0}^{1} \tau^{\beta-1} d H(\tau)\right]=\sigma_{1} h_{1}(s) .
\end{aligned}
$$

$\left(\mathrm{a}_{2}\right)$

$$
\begin{aligned}
G_{1}(t, s) & \leq \frac{t^{\alpha-1}}{\Gamma(\alpha-1)}+\frac{t^{\alpha-1}}{\Delta}\left(\int_{0}^{1} \tau^{\beta-1} d H(\tau)\right)\left(\int_{0}^{1} \frac{(1-\tau) \tau^{\alpha-1}}{\Gamma(\alpha-1)} d K(\tau)\right) \\
& =t^{\alpha-1} \frac{1}{\Gamma(\alpha-1)}\left[1+\frac{1}{\Delta}\left(\int_{0}^{1} \tau^{\beta-1} d H(\tau)\right)\left(\int_{0}^{1}(1-\tau) \tau^{\alpha-1} d K(\tau)\right)\right]=\delta_{1} t^{\alpha-1} .
\end{aligned}
$$

$\left(a_{3}\right)$

$$
\begin{aligned}
G_{1}(t, s) & \geq \frac{t^{\alpha-1}}{\Delta}\left(\int_{0}^{1} \tau^{\beta-1} d H(\tau)\right)\left(\int_{0}^{1} k_{1}(\tau) h_{1}(s) d K(\tau)\right) \\
& =t^{\alpha-1} h_{1}(s) \frac{1}{\Delta}\left(\int_{0}^{1} \tau^{\beta-1} d H(\tau)\right)\left(\int_{0}^{1} k_{1}(\tau) d K(\tau)\right)=\varrho_{1} t^{\alpha-1} h_{1}(s) .
\end{aligned}
$$


$\left(b_{1}\right)$

$$
\begin{aligned}
G_{2}(t, s) & =\frac{t^{\alpha-1}}{\Delta} \int_{0}^{1} g_{2}(\tau, s) d H(\tau) \leq \frac{1}{\Delta} \int_{0}^{1} h_{2}(s) d H(\tau) \\
& =\frac{1}{\Delta}(H(1)-H(0)) h_{2}(s)=\sigma_{2} h_{2}(s) .
\end{aligned}
$$

$\left(b_{2}\right)$

$$
G_{2}(t, s) \leq \frac{t^{\alpha-1}}{\Delta} \int_{0}^{1} \frac{(1-\tau) \tau^{\beta-1}}{\Gamma(\beta-1)} d H(\tau)=\delta_{2} t^{\alpha-1} .
$$

$\left(b_{3}\right)$

$$
G_{2}(t, s) \geq \frac{t^{\alpha-1}}{\Delta} \int_{0}^{1} k_{2}(\tau) h_{2}(s) d H(\tau)=\frac{t^{\alpha-1}}{\Delta} h_{2}(s) \int_{0}^{1} k_{2}(\tau) d H(\tau)=\varrho_{2} t^{\alpha-1} h_{2}(s) .
$$

$\left(c_{1}\right)$

$$
\begin{aligned}
G_{3}(t, s) & =g_{2}(t, s)+\frac{t^{\beta-1}}{\Delta}\left(\int_{0}^{1} \tau^{\alpha-1} d K(\tau)\right)\left(\int_{0}^{1} g_{2}(\tau, s) d H(\tau)\right) \\
& \leq h_{2}(s)+\frac{1}{\Delta}\left(\int_{0}^{1} \tau^{\alpha-1} d K(\tau)\right)\left(\int_{0}^{1} h_{2}(s) d H(\tau)\right) \\
& =h_{2}(s)\left[1+\frac{1}{\Delta}(H(1)-H(0)) \int_{0}^{1} \tau^{\alpha-1} d K(\tau)\right]=\sigma_{3} h_{2}(s) .
\end{aligned}
$$

$\left(c_{2}\right)$

$$
\begin{aligned}
G_{3}(t, s) & \leq \frac{(1-t) t^{\beta-1}}{\Gamma(\beta-1)}+\frac{t^{\beta-1}}{\Delta}\left(\int_{0}^{1} \tau^{\alpha-1} d K(\tau)\right)\left(\int_{0}^{1} \frac{(1-\tau) \tau^{\beta-1}}{\Gamma(\beta-1)} d H(\tau)\right) \\
& \leq \frac{t^{\beta-1}}{\Gamma(\beta-1)}\left[1+\frac{1}{\Delta}\left(\int_{0}^{1} \tau^{\alpha-1} d K(\tau)\right)\left(\int_{0}^{1}(1-\tau) \tau^{\beta-1} d H(\tau)\right)\right]=\delta_{3} t^{\beta-1} .
\end{aligned}
$$

$\left(c_{3}\right)$

$$
\begin{aligned}
G_{3}(t, s) & \geq \frac{t^{\beta-1}}{\Delta}\left(\int_{0}^{1} \tau^{\alpha-1} d K(\tau)\right)\left(\int_{0}^{1} k_{2}(\tau) h_{2}(s) d H(\tau)\right) \\
& =t^{\beta-1} h_{2}(s) \frac{1}{\Delta}\left(\int_{0}^{1} \tau^{\alpha-1} d K(\tau)\right)\left(\int_{0}^{1} k_{2}(\tau) d H(\tau)\right)=\varrho_{3} t^{\beta-1} h_{2}(s) .
\end{aligned}
$$

$\left(d_{1}\right)$

$$
\begin{aligned}
G_{4}(t, s) & =\frac{t^{\beta-1}}{\Delta} \int_{0}^{1} g_{1}(\tau, s) d K(\tau) \leq \frac{1}{\Delta} \int_{0}^{1} h_{1}(s) d K(\tau) \\
& =h_{1}(s) \frac{1}{\Delta}(K(1)-K(0))=\sigma_{4} h_{1}(s) .
\end{aligned}
$$

$\left(d_{2}\right)$

$$
G_{4}(t, s) \leq \frac{t^{\beta-1}}{\Delta} \int_{0}^{1} \frac{(1-\tau) \tau^{\alpha-1}}{\Gamma(\alpha-1)} d K(\tau)=\delta_{4} t^{\beta-1} .
$$


$\left(d_{3}\right)$

$$
G_{4}(t, s) \geq \frac{t^{\beta-1}}{\Delta} \int_{0}^{1} k_{1}(\tau) h_{1}(s) d K(\tau)=t^{\beta-1} h_{1}(s) \frac{1}{\Delta} \int_{0}^{1} k_{1}(s) d K(\tau)=\varrho_{4} t^{\beta-1} h_{1}(s) .
$$

Lemma 2.5 Assume that $H, K:[0,1] \rightarrow \mathbb{R}$ are nondecreasing functions, $\Delta>0, \int_{0}^{1} \tau^{\alpha-1}(1-$ $\tau) d K(\tau)>0, \int_{0}^{1} \tau^{\beta-1}(1-\tau) d H(\tau)>0$, and $\tilde{x}, \tilde{y} \in C(0,1) \cap L^{1}(0,1), \tilde{x}(t) \geq 0, \tilde{y}(t) \geq 0$ for all $t \in(0,1)$. Then the solution $(u, v)$ of problem (1)-(2) given by (3) satisfies the inequalities $u(t) \geq \gamma_{1} t^{\alpha-1} u\left(t^{\prime}\right), v(t) \geq \gamma_{2} t^{\beta-1} v\left(t^{\prime}\right)$, for all $t, t^{\prime} \in[0,1]$, where $\gamma_{1}=\min \left\{\frac{\varrho_{1}}{\sigma_{1}}, \frac{\varrho_{2}}{\sigma_{2}}\right\}>0, \gamma_{2}=$ $\min \left\{\frac{\varrho_{3}}{\sigma_{3}}, \frac{\varrho 4}{\sigma_{4}}\right\}>0$.

Proof By using Lemma 2.4, we obtain

$$
\begin{aligned}
u(t) & =\int_{0}^{1} G_{1}(t, s) \tilde{x}(s) d s+\int_{0}^{1} G_{2}(t, s) \tilde{y}(s) d s \\
& \geq \int_{0}^{1} \varrho_{1} t^{\alpha-1} h_{1}(s) \tilde{x}(s) d s+\int_{0}^{1} \varrho_{2} t^{\alpha-1} h_{2}(s) \tilde{y}(s) d s \\
& =t^{\alpha-1}\left(\varrho_{1} \int_{0}^{1} h_{1}(s) \tilde{x}(s) d s+\varrho_{2} \int_{0}^{1} h_{2}(s) \tilde{y}(s) d s\right) \\
& \geq t^{\alpha-1}\left(\frac{\varrho_{1}}{\sigma_{1}} \int_{0}^{1} G_{1}\left(t^{\prime}, s\right) \tilde{x}(s) d s+\frac{\varrho_{2}}{\sigma_{2}} \int_{0}^{1} G_{2}\left(t^{\prime}, s\right) \tilde{y}(s) d s\right) \\
& \geq t^{\alpha-1} \min \left\{\frac{\varrho_{1}}{\sigma_{1}}, \frac{\varrho_{2}}{\sigma_{2}}\right\}\left(\int_{0}^{1} G_{1}\left(t^{\prime}, s\right) \tilde{x}(s) d s+\int_{0}^{1} G_{2}\left(t^{\prime}, s\right) \tilde{y}(s) d s\right) \\
& =\gamma_{1} t^{\alpha-1} u\left(t^{\prime}\right), \quad \forall t, t^{\prime} \in[0,1], \text { where } \gamma_{1}=\min \left\{\frac{\varrho_{1}}{\sigma_{1}}, \frac{\varrho_{2}}{\sigma_{2}}\right\}>0 .
\end{aligned}
$$

In a similar way, we deduce

$$
\begin{aligned}
v(t) & =\int_{0}^{1} G_{3}(t, s) \tilde{y}(s) d s+\int_{0}^{1} G_{4}(t, s) \tilde{x}(s) d s \\
& \geq \gamma_{2} t^{\beta-1} v\left(t^{\prime}\right), \quad \forall t, t^{\prime} \in[0,1], \text { where } \gamma_{2}=\min \left\{\frac{\varrho_{3}}{\sigma_{3}}, \frac{\varrho_{4}}{\sigma_{4}}\right\}>0 .
\end{aligned}
$$

In the proof of our main results we shall use the nonlinear alternative of Leray-Schauder type and the Guo-Krasnosel'skii fixed point theorem presented below (see [14, 15]).

Theorem 2.1 Let X be a Banach space with $\Omega \subset X$ closed and convex. Assume $U$ is a relatively open subset of $\Omega$ with $0 \in U$, and let $S: \bar{U} \rightarrow \Omega$ be a completely continuous operator (continuous and compact). Then either

(1) S has a fixed point in $\bar{U}$, or

(2) there exist $u \in \partial U$ and $v \in(0,1)$ such that $u=v S u$.

Theorem 2.2 Let $X$ be a Banach space and let $C \subset X$ be a cone in $X$. Assume $\Omega_{1}$ and $\Omega_{2}$ are bounded open subsets of $X$ with $0 \in \Omega_{1} \subset \bar{\Omega}_{1} \subset \Omega_{2}$ and let $\mathcal{A}: C \cap\left(\bar{\Omega}_{2} \backslash \Omega_{1}\right) \rightarrow C$ be a completely continuous operator such that either

(i) $\|\mathcal{A} u\| \leq\|u\|, u \in C \cap \partial \Omega_{1}$, and $\|\mathcal{A} u\| \geq\|u\|, u \in C \cap \partial \Omega_{2}$, or

(ii) $\|\mathcal{A} u\| \geq\|u\|, u \in C \cap \partial \Omega_{1}$, and $\|\mathcal{A} u\| \leq\|u\|, u \in C \cap \partial \Omega_{2}$.

Then $\mathcal{A}$ has a fixed point in $C \cap\left(\bar{\Omega}_{2} \backslash \Omega_{1}\right)$. 


\section{Main results}

In this section, we investigate the existence and multiplicity of positive solutions for our problem $(\mathrm{S})-(\mathrm{BC})$. We present now the assumptions that we shall use in the sequel.

(H1) $H, K:[0,1] \rightarrow \mathbb{R}$ are nondecreasing functions, $\Delta=1-\left(\int_{0}^{1} \tau^{\alpha-1} d K(\tau)\right) \times$ $\left(\int_{0}^{1} \tau^{\beta-1} d H(\tau)\right)>0$, and $\int_{0}^{1} \tau^{\alpha-1}(1-\tau) d K(\tau)>0, \int_{0}^{1} \tau^{\beta-1}(1-\tau) d H(\tau)>0$.

(H2) The functions $f, g \in C([0,1] \times[0, \infty) \times[0, \infty),(-\infty,+\infty))$ and there exist functions $p_{1}, p_{2} \in C([0,1],[0, \infty))$ such that $f(t, u, v) \geq-p_{1}(t)$ and $g(t, u, v) \geq-p_{2}(t)$ for any $t \in$ $[0,1]$ and $u, v \in[0, \infty)$.

(H3) $f(t, 0,0)>0, g(t, 0,0)>0$ for all $t \in[0,1]$.

(H4) The functions $f, g \in C((0,1) \times[0, \infty) \times[0, \infty),(-\infty,+\infty)), f, g$ may be singular at $t=0$ and/or $t=1$, and there exist functions $p_{1}, p_{2} \in C((0,1),[0, \infty)), \alpha_{1}, \alpha_{2} \in$ $C((0,1),[0, \infty)), \beta_{1}, \beta_{2} \in C([0,1] \times[0, \infty) \times[0, \infty),[0, \infty))$ such that

$$
-p_{1}(t) \leq f(t, u, v) \leq \alpha_{1}(t) \beta_{1}(t, u, v), \quad-p_{2}(t) \leq g(t, u, v) \leq \alpha_{2}(t) \beta_{2}(t, u, v)
$$

for all $t \in(0,1), u, v \in[0, \infty)$, with $0<\int_{0}^{1} p_{i}(s) d s<\infty, 0<\int_{0}^{1} \alpha_{i}(s) d s<\infty, i=1,2$.

(H5) There exists $c \in(0,1 / 2)$ such that

$$
f_{\infty}=\lim _{u+v \rightarrow \infty} \min _{t \in[c, 1-c]} \frac{f(t, u, v)}{u+v}=\infty \quad \text { or } \quad g_{\infty}=\lim _{u+v \rightarrow \infty} \min _{t \in[c, 1-c]} \frac{g(t, u, v)}{u+v}=\infty .
$$

(H6) $\beta_{i \infty}=\lim _{u+v \rightarrow \infty} \max _{t \in[0,1]} \frac{\beta_{i}(t, u, v)}{u+v}=0, i=1,2$.

We consider the system of nonlinear fractional differential equations

$$
\begin{cases}D_{0+}^{\alpha} x(t)+\lambda\left(f\left(t,\left[x(t)-q_{1}(t)\right]^{*},\left[y(t)-q_{2}(t)\right]^{*}\right)+p_{1}(t)\right)=0, & 0<t<1, \\ D_{0+}^{\beta} y(t)+\mu\left(g\left(t,\left[x(t)-q_{1}(t)\right]^{*},\left[y(t)-q_{2}(t)\right]^{*}\right)+p_{2}(t)\right)=0, & 0<t<1,\end{cases}
$$

with the integral boundary conditions

$$
\begin{cases}x(0)=x^{\prime}(0)=\cdots=x^{(n-2)}(0)=0, & x(1)=\int_{0}^{1} y(s) d H(s), \\ y(0)=y^{\prime}(0)=\cdots=y^{(m-2)}(0)=0, & y(1)=\int_{0}^{1} x(s) d K(s),\end{cases}
$$

where $z(t)^{*}=z(t)$ if $z(t) \geq 0$, and $z(t)^{*}=0$ if $z(t)<0$. Here $\left(q_{1}, q_{2}\right)$ with

$$
\begin{array}{ll}
q_{1}(t)=\lambda \int_{0}^{1} G_{1}(t, s) p_{1}(s) d s+\mu \int_{0}^{1} G_{2}(t, s) p_{2}(s) d s, & t \in[0,1], \\
q_{2}(t)=\mu \int_{0}^{1} G_{3}(t, s) p_{2}(s) d s+\lambda \int_{0}^{1} G_{4}(t, s) p_{1}(s) d s, & t \in[0,1],
\end{array}
$$

is solution of the system of fractional differential equations

$$
\left\{\begin{array}{l}
D_{0+}^{\alpha} q_{1}(t)+\lambda p_{1}(t)=0, \quad 0<t<1 \\
D_{0+}^{\beta} q_{2}(t)+\mu p_{2}(t)=0, \quad 0<t<1
\end{array}\right.
$$

with the integral boundary conditions

$$
\left\{\begin{array}{lr}
q_{1}(0)=q_{1}^{\prime}(0)=\cdots=q_{1}^{(n-2)}(0)=0, & q_{1}(1)=\int_{0}^{1} q_{2}(s) d H(s), \\
q_{2}(0)=q_{2}^{\prime}(0)=\cdots=q_{2}^{(m-2)}(0)=0, & q_{2}(1)=\int_{0}^{1} q_{1}(s) d K(s) .
\end{array}\right.
$$


Under the assumptions (H1) and (H2), or (H1) and (H4), we have $q_{1}(t) \geq 0, q_{2}(t) \geq 0$ for all $t \in[0,1]$.

We shall prove that there exists a solution $(x, y)$ for the boundary value problem (6)-(7) with $x(t) \geq q_{1}(t)$ and $y(t) \geq q_{2}(t)$ on $[0,1], x(t)>q_{1}(t), y(t)>q_{2}(t)$ on $(0,1)$. In this case $(u, v)$ with $u(t)=x(t)-q_{1}(t)$ and $v(t)=y(t)-q_{2}(t), t \in[0,1]$ represents a positive solution of boundary value problem $(\mathrm{S})-(\mathrm{BC})$.

By using Lemma 2.1 (relations (3)), a solution of the system

$$
\left\{\begin{aligned}
x(t)= & \lambda \int_{0}^{1} G_{1}(t, s)\left(f\left(s,\left[x(s)-q_{1}(s)\right]^{*},\left[y(s)-q_{2}(s)\right]^{*}\right)+p_{1}(s)\right) d s \\
& +\mu \int_{0}^{1} G_{2}(t, s)\left(g\left(s,\left[x(s)-q_{1}(s)\right]^{*},\left[y(s)-q_{2}(s)\right]^{*}\right)+p_{2}(s)\right) d s, \quad t \in[0,1], \\
y(t)= & \mu \int_{0}^{1} G_{3}(t, s)\left(g\left(s,\left[x(s)-q_{1}(s)\right]^{*},\left[y(s)-q_{2}(s)\right]^{*}\right)+p_{2}(s)\right) d s \\
& +\lambda \int_{0}^{1} G_{4}(t, s)\left(f\left(s,\left[x(s)-q_{1}(s)\right]^{*},\left[y(s)-q_{2}(s)\right]^{*}\right)+p_{1}(s)\right) d s, \quad t \in[0,1],
\end{aligned}\right.
$$

is a solution for problem (6)-(7).

We consider the Banach space $X=C([0,1])$ with the supremum norm $\|\cdot\|$, and the Banach space $Y=X \times X$ with the norm $\|(u, v)\|_{Y}=\|u\|+\|v\|$. We define the cones

$$
\begin{aligned}
& P_{1}=\left\{x \in X, x(t) \geq \gamma_{1} t^{\alpha-1}\|x\|, \forall t \in[0,1]\right\}, \\
& P_{2}=\left\{y \in X, y(t) \geq \gamma_{2} t^{\beta-1}\|y\|, \forall t \in[0,1]\right\},
\end{aligned}
$$

where $\gamma_{1}, \gamma_{2}$ are defined in Section 2 (Lemma 2.5), and $P=P_{1} \times P_{2} \subset Y$.

For $\lambda, \mu>0$, we introduce the operators $Q_{1}, Q_{2}: Y \rightarrow X$ and $\mathcal{Q}: Y \rightarrow Y$ defined by $\mathcal{Q}(x, y)=\left(Q_{1}(x, y), Q_{2}(x, y)\right),(x, y) \in Y$ with

$$
\begin{aligned}
Q_{1}(x, y)(t)= & \lambda \int_{0}^{1} G_{1}(t, s)\left(f\left(s,\left[x(s)-q_{1}(s)\right]^{*},\left[y(s)-q_{2}(s)\right]^{*}\right)+p_{1}(s)\right) d s \\
& +\mu \int_{0}^{1} G_{2}(t, s)\left(g\left(s,\left[x(s)-q_{1}(s)\right]^{*},\left[y(s)-q_{2}(s)\right]^{*}\right)+p_{2}(s)\right) d s, \quad t \in[0,1], \\
Q_{2}(x, y)(t)= & \mu \int_{0}^{1} G_{3}(t, s)\left(g\left(s,\left[x(s)-q_{1}(s)\right]^{*},\left[y(s)-q_{2}(s)\right]^{*}\right)+p_{2}(s)\right) d s \\
& +\lambda \int_{0}^{1} G_{4}(t, s)\left(f\left(s,\left[x(s)-q_{1}(s)\right]^{*},\left[y(s)-q_{2}(s)\right]^{*}\right)+p_{1}(s)\right) d s, \quad t \in[0,1] .
\end{aligned}
$$

It is clear that if $(x, y)$ is a fixed point of operator $\mathcal{Q}$, then $(x, y)$ is a solution of problem (6)-(7).

Lemma 3.1 If $(\mathrm{H} 1)$ and $(\mathrm{H} 2)$, or $(\mathrm{H} 1)$ and $(\mathrm{H} 4)$ hold, then operator $\mathcal{Q}: P \rightarrow P$ is a completely continuous operator.

Proof The operators $Q_{1}$ and $Q_{2}$ are well defined. To prove this, let $(x, y) \in P$ be fixed with $\|(x, y)\|_{Y}=\widetilde{L}$. Then we have

$$
\begin{array}{ll}
{\left[x(s)-q_{1}(s)\right]^{*} \leq x(s) \leq\|x\| \leq\|(x, y)\|_{Y}=\widetilde{L},} & \forall s \in[0,1], \\
{\left[y(s)-q_{2}(s)\right]^{*} \leq y(s) \leq\|y\| \leq\|(x, y)\|_{Y}=\widetilde{L},} & \forall s \in[0,1] .
\end{array}
$$

If (H1) and (H2) hold, then we deduce easily that $Q_{1}(x, y)(t)<\infty$ and $Q_{2}(x, y)(t)<\infty$ for all $t \in[0,1]$. 
If (H1) and (H4) hold, we deduce, for all $t \in[0,1]$,

$$
\begin{aligned}
Q_{1}(x, y)(t) \leq & \lambda \sigma_{1} \int_{0}^{1} h_{1}(s)\left[\alpha_{1}(s) \beta_{1}\left(s,\left[x(s)-q_{1}(s)\right]^{*},\left[y(s)-q_{2}(s)\right]^{*}\right)+p_{1}(s)\right] d s \\
& +\mu \sigma_{2} \int_{0}^{1} h_{2}(s)\left[\alpha_{2}(s) \beta_{2}\left(s,\left[x(s)-q_{1}(s)\right]^{*},\left[y(s)-q_{2}(s)\right]^{*}\right)+p_{2}(s)\right] d s \\
\leq & M\left(\lambda \sigma_{1} \int_{0}^{1} h_{1}(s)\left(\alpha_{1}(s)+p_{1}(s)\right) d s+\mu \sigma_{2} \int_{0}^{1} h_{2}(s)\left(\alpha_{2}(s)+p_{2}(s)\right) d s\right)<\infty \\
Q_{2}(x, y)(t) \leq & \mu \sigma_{3} \int_{0}^{1} h_{2}(s)\left[\alpha_{2}(s) \beta_{2}\left(s,\left[x(s)-q_{1}(s)\right]^{*},\left[y(s)-q_{2}(s)\right]^{*}\right)+p_{2}(s)\right] d s \\
& +\lambda \sigma_{4} \int_{0}^{1} h_{1}(s)\left[\alpha_{1}(s) \beta_{1}\left(s,\left[x(s)-q_{1}(s)\right]^{*},\left[y(s)-q_{2}(s)\right]^{*}\right)+p_{1}(s)\right] d s \\
\leq & M\left(\mu \sigma_{3} \int_{0}^{1} h_{2}(s)\left(\alpha_{2}(s)+p_{2}(s)\right) d s+\lambda \sigma_{4} \int_{0}^{1} h_{1}(s)\left(\alpha_{1}(s)+p_{1}(s)\right) d s\right)<\infty
\end{aligned}
$$

where $M=\max \left\{\max _{t \in[0,1], u, v \in[0, \widetilde{L}]} \beta_{1}(t, u, v), \max _{t \in[0,1], u, v \in[0, \widetilde{L}]} \beta_{2}(t, u, v), 1\right\}$.

Besides, by Lemma 2.5, we conclude that

$$
Q_{1}(x, y)(t) \geq \gamma_{1} t^{\alpha-1}\left\|Q_{1}(x, y)\right\|, \quad Q_{2}(x, y)(t) \geq \gamma_{2} t^{\beta-1}\left\|Q_{2}(x, y)\right\|, \quad \forall t \in[0,1],
$$

and so $Q_{1}(x, y), Q_{2}(x, y) \in P$.

By using standard arguments, we deduce that operator $\mathcal{Q}: P \rightarrow P$ is a completely continuous operator (a compact operator, that is, one that maps bounded sets into relatively compact sets and is continuous).

Theorem 3.1 Assume that (H1)-(H3) hold. Then there exist constants $\lambda_{0}>0$ and $\mu_{0}>0$ such that, for any $\lambda \in\left(0, \lambda_{0}\right]$ and $\mu \in\left(0, \mu_{0}\right]$, the boundary value problem (S)-(BC) has at least one positive solution.

Proof Let $\delta \in(0,1)$ be fixed. From (H2) and (H3), there exists $R_{0} \in(0,1]$ such that

$$
f(t, u, v) \geq \delta f(t, 0,0), \quad g(t, u, v) \geq \delta g(t, 0,0), \quad \forall t \in[0,1], u, v \in\left[0, R_{0}\right]
$$

We define

$$
\begin{aligned}
& \bar{f}\left(R_{0}\right)=\max _{t \in[0,1], u, v \in\left[0, R_{0}\right]}\left\{f(t, u, v)+p_{1}(t)\right\} \geq \max _{t \in[0,1]}\left\{\delta f(t, 0,0)+p_{1}(t)\right\}>0, \\
& \bar{g}\left(R_{0}\right)=\max _{t \in[0,1], u, v \in\left[0, R_{0}\right]}\left\{g(t, u, v)+p_{2}(t)\right\} \geq \max _{t \in[0,1]}\left\{\delta g(t, 0,0)+p_{2}(t)\right\}>0, \\
& c_{1}=\sigma_{1} \int_{0}^{1} h_{1}(s) d s, \quad c_{2}=\sigma_{2} \int_{0}^{1} h_{2}(s) d s, \\
& c_{3}=\sigma_{3} \int_{0}^{1} h_{2}(s) d s, \quad c_{4}=\sigma_{4} \int_{0}^{1} h_{1}(s) d s, \\
& \lambda_{0}=\max \left\{\frac{R_{0}}{8 c_{1} \bar{f}\left(R_{0}\right)}, \frac{R_{0}}{8 c_{4} \bar{f}\left(R_{0}\right)}\right\}, \quad \mu_{0}=\max \left\{\frac{R_{0}}{8 c_{2} \bar{g}\left(R_{0}\right)}, \frac{R_{0}}{8 c_{3} \bar{g}\left(R_{0}\right)}\right\} .
\end{aligned}
$$


We will show that, for any $\lambda \in\left(0, \lambda_{0}\right]$ and $\mu \in\left(0, \mu_{0}\right]$, problem (6)-(7) has at least one positive solution.

So, let $\lambda \in\left(0, \lambda_{0}\right]$ and $\mu \in\left(0, \mu_{0}\right]$ be arbitrary, but fixed for the moment. We define the set $U=\left\{(x, y) \in P,\|(u, v)\|_{Y}<R_{0}\right\}$. We suppose that there exist $(x, y) \in \partial U\left(\|(x, y)\|_{Y}=R_{0}\right.$ or $\left.\|x\|+\|y\|=R_{0}\right)$ and $v \in(0,1)$ such that $(x, y)=v \mathcal{Q}(x, y)$ or $x=v Q_{1}(x, y), y=v Q_{2}(x, y)$.

We deduce that

$$
\begin{aligned}
& {\left[x(t)-q_{1}(t)\right]^{*}=x(t)-q_{1}(t) \leq x(t) \leq R_{0}, \quad \text { if } x(t)-q_{1}(t) \geq 0,} \\
& {\left[x(t)-q_{1}(t)\right]^{*}=0, \quad \text { for } x(t)-q_{1}(t)<0, \forall t \in[0,1],} \\
& {\left[y(t)-q_{2}(t)\right]^{*}=y(t)-q_{2}(t) \leq y(t) \leq R_{0}, \quad \text { if } y(t)-q_{2}(t) \geq 0,} \\
& {\left[y(t)-q_{2}(t)\right]^{*}=0, \quad \text { for } y(t)-q_{2}(t)<0, \forall t \in[0,1] .}
\end{aligned}
$$

Then by Lemma 2.4 , for all $t \in[0,1]$, we obtain

$$
\begin{aligned}
x(t) & =\nu Q_{1}(x, y)(t) \leq Q_{1}(x, y)(t) \\
& \leq \lambda \sigma_{1} \int_{0}^{1} h_{1}(s) \bar{f}\left(R_{0}\right) d s+\mu \sigma_{2} \int_{0}^{1} h_{2}(s) \bar{g}\left(R_{0}\right) d s \\
& \leq \lambda_{0} c_{1} \bar{f}\left(R_{0}\right)+\mu_{0} c_{2} \bar{g}\left(R_{0}\right) \leq \frac{R_{0}}{8}+\frac{R_{0}}{8}=\frac{R_{0}}{4}, \\
y(t) & =\nu Q_{2}(x, y)(t) \leq Q_{2}(x, y)(t) \\
& \leq \mu \sigma_{3} \int_{0}^{1} h_{2}(s) \bar{g}\left(R_{0}\right) d s+\lambda \sigma_{4} \int_{0}^{1} h_{1}(s) \bar{f}\left(R_{0}\right) d s \\
& \leq \mu_{0} c_{3} \bar{g}\left(R_{0}\right)+\lambda_{0} c_{4} \bar{f}\left(R_{0}\right) \leq \frac{R_{0}}{8}+\frac{R_{0}}{8}=\frac{R_{0}}{4} .
\end{aligned}
$$

Hence $\|x\| \leq \frac{R_{0}}{4}$ and $\|y\| \leq \frac{R_{0}}{4}$. Then $R_{0}=\|(x, y)\|_{Y}=\|x\|+\|y\| \leq \frac{R_{0}}{4}+\frac{R_{0}}{4}=\frac{R_{0}}{2}$, which is a contradiction.

Therefore, by Theorem 2.1 (with $\Omega=P$ ), we deduce that $\mathcal{Q}$ has a fixed point $\left(x_{0}, y_{0}\right) \in$ $\bar{U} \cap P$. That is, $\left(x_{0}, y_{0}\right)=\mathcal{Q}\left(x_{0}, y_{0}\right)$ or $x_{0}=Q_{1}\left(x_{0}, y_{0}\right), y_{0}=Q_{2}\left(x_{0}, y_{0}\right)$, and $\left\|x_{0}\right\|+\left\|y_{0}\right\| \leq R_{0}$ with $x_{0}(t) \geq \gamma_{1} t^{\alpha-1}\left\|x_{0}\right\|$ and $y_{0}(t) \geq \gamma_{2} t^{\beta-1}\left\|y_{0}\right\|$ for all $t \in[0,1]$.

Moreover, by (10), we conclude

$$
\begin{aligned}
x_{0}(t) & =Q_{1}\left(x_{0}, y_{0}\right)(t) \\
& \geq \lambda \int_{0}^{1} G_{1}(t, s)\left(\delta f(t, 0,0)+p_{1}(s)\right) d s+\mu \int_{0}^{1} G_{2}(t, s)\left(\delta g(t, 0,0)+p_{2}(s)\right) d s \\
& \geq \lambda \int_{0}^{1} G_{1}(t, s) p_{1}(s) d s+\mu \int_{0}^{1} G_{2}(t, s) p_{2}(s) d s=q_{1}(t), \quad \forall t \in[0,1], \\
x_{0}(t) & >\lambda \int_{0}^{1} G_{1}(t, s) p_{1}(s) d s+\mu \int_{0}^{1} G_{2}(t, s) p_{2}(s) d s=q_{1}(t), \quad \forall t \in(0,1), \\
y_{0}(t) & =Q_{2}\left(x_{0}, y_{0}\right)(t) \\
& \geq \mu \int_{0}^{1} G_{3}(t, s)\left(\delta g(t, 0,0)+p_{2}(s)\right) d s+\lambda \int_{0}^{1} G_{4}(t, s)\left(\delta f(t, 0,0)+p_{1}(s)\right) d s
\end{aligned}
$$




$$
\begin{aligned}
\geq \mu \int_{0}^{1} G_{3}(t, s) p_{2}(s) d s+\lambda \int_{0}^{1} G_{4}(t, s) p_{1}(s) d s=q_{2}(t), \quad \forall t \in[0,1], \\
y_{0}(t)>\mu \int_{0}^{1} G_{3}(t, s) p_{2}(s) d s+\lambda \int_{0}^{1} G_{4}(t, s) p_{1}(s) d s=q_{2}(t), \quad \forall t \in(0,1) .
\end{aligned}
$$

Therefore $x_{0}(t) \geq q_{1}(t), y_{0}(t) \geq q_{2}(t)$ for all $t \in[0,1]$, and $x_{0}(t)>q_{1}(t), y_{0}(t)>q_{2}(t)$ for all $t \in(0,1)$. Let $u_{0}(t)=x_{0}(t)-q_{1}(t)$ and $v_{0}(t)=y_{0}(t)-q_{2}(t)$ for all $t \in[0,1]$. Then $u_{0}(t) \geq 0$, $v_{0}(t) \geq 0$ for all $t \in[0,1], u_{0}(t)>0, v_{0}(t)>0$ for all $t \in(0,1)$. Therefore $\left(u_{0}, v_{0}\right)$ is a positive solution of (S)-(BC).

Theorem 3.2 Assume that (H1), (H4), and (H5) hold. Then there exist $\lambda^{*}>0$ and $\mu^{*}>0$ such that, for any $\lambda \in\left(0, \lambda^{*}\right]$ and $\mu \in\left(0, \mu^{*}\right]$, the boundary value problem (S)-(BC) has at least one positive solution.

Proof We choose a positive number

$$
\begin{aligned}
R_{1}> & \max \left\{1, \frac{2}{\gamma_{1}} \int_{0}^{1}\left(\delta_{1} p_{1}(s)+\delta_{2} p_{2}(s)\right) d s, \frac{2}{\gamma_{2}} \int_{0}^{1}\left(\delta_{3} p_{2}(s)+\delta_{4} p_{1}(s)\right) d s,\right. \\
& \frac{2}{\gamma_{1} \gamma_{2}}\left(\int_{0}^{1} s^{\beta-1} d H(s)\right)^{-1} \int_{0}^{1}\left(\delta_{1} p_{1}(s)+\delta_{2} p_{2}(s)\right) d s, \\
& \left.\frac{2}{\gamma_{1} \gamma_{2}}\left(\int_{0}^{1} s^{\alpha-1} d K(s)\right)^{-1} \int_{0}^{1}\left(\delta_{3} p_{2}(s)+\delta_{4} p_{1}(s)\right) d s\right\},
\end{aligned}
$$

and we define the set $\Omega_{1}=\left\{(x, y) \in P,\|(x, y)\|_{Y}<R_{1}\right\}$.

We introduce

$$
\begin{aligned}
\lambda^{*}= & \min \left\{1, \frac{R_{1}}{4 \sigma_{1} M_{1}}\left(\int_{0}^{1} h_{1}(s)\left(\alpha_{1}(s)+p_{1}(s)\right) d s\right)^{-1},\right. \\
& \left.\frac{R_{1}}{4 \sigma_{4} M_{1}}\left(\int_{0}^{1} h_{1}(s)\left(\alpha_{1}(s)+p_{1}(s)\right) d s\right)^{-1}\right\}, \\
\mu^{*}= & \min \left\{1, \frac{R_{1}}{4 \sigma_{2} M_{2}}\left(\int_{0}^{1} h_{2}(s)\left(\alpha_{2}(s)+p_{2}(s)\right) d s\right)^{-1},\right. \\
& \left.\frac{R_{1}}{4 \sigma_{3} M_{2}}\left(\int_{0}^{1} h_{2}(s)\left(\alpha_{2}(s)+p_{2}(s)\right) d s\right)^{-1}\right\},
\end{aligned}
$$

with

$$
\begin{aligned}
& M_{1}=\max \left\{\max _{\substack{t \in[0,1] \\
u, v \geq 0, u+v \leq R_{1}}} \beta_{1}(t, u, v), 1\right\}, \\
& M_{2}=\max \left\{\max _{\substack{t \in[0,1] \\
u, v \geq 0, u+v \leq R_{1}}} \beta_{2}(t, u, v), 1\right\} .
\end{aligned}
$$

Let $\lambda \in\left(0, \lambda^{*}\right]$ and $\mu \in\left(0, \mu^{*}\right]$. Then, for any $(x, y) \in P \cap \partial \Omega_{1}$ and $s \in[0,1]$, we have

$$
\begin{aligned}
& {\left[x(s)-q_{1}(s)\right]^{*} \leq x(s) \leq\|x\| \leq R_{1},} \\
& {\left[y(s)-q_{2}(s)\right]^{*} \leq y(s) \leq\|y\| \leq R_{1} .}
\end{aligned}
$$


Then, for any $(x, y) \in P \cap \partial \Omega_{1}$, we obtain

$$
\begin{aligned}
\left\|Q_{1}(x, y)\right\| \leq & \lambda \sigma_{1} \int_{0}^{1} h_{1}(s)\left[\alpha_{1}(s) \beta_{1}\left(s,\left[x(s)-q_{1}(s)\right]^{*},\left[y(s)-q_{2}(s)\right]^{*}\right)+p_{1}(s)\right] d s \\
& +\mu \sigma_{2} \int_{0}^{1} h_{2}(s)\left[\alpha_{2}(s) \beta_{2}\left(s,\left[x(s)-q_{1}(s)\right]^{*},\left[y(s)-q_{2}(s)\right]^{*}\right)+p_{2}(s)\right] d s \\
\leq & \lambda^{*} \sigma_{1} M_{1} \int_{0}^{1} h_{1}(s)\left(\alpha_{1}(s)+p_{1}(s)\right) d s+\mu^{*} \sigma_{2} M_{2} \int_{0}^{1} h_{2}(s)\left(\alpha_{2}(s)+p_{2}(s)\right) d s \\
\leq & \frac{R_{1}}{4}+\frac{R_{1}}{4}=\frac{R_{1}}{2}=\frac{\|(x, y)\|_{Y}}{2}, \\
\left\|Q_{2}(x, y)\right\| \leq & \mu \sigma_{3} \int_{0}^{1} h_{2}(s)\left[\alpha_{2}(s) \beta_{2}\left(s,\left[x(s)-q_{1}(s)\right]^{*},\left[y(s)-q_{2}(s)\right]^{*}\right)+p_{2}(s)\right] d s \\
& +\lambda \sigma_{4} \int_{0}^{1} h_{1}(s)\left[\alpha_{1}(s) \beta_{1}\left(s,\left[x(s)-q_{1}(s)\right]^{*},\left[y(s)-q_{2}(s)\right]^{*}\right)+p_{1}(s)\right] d s \\
\leq & \mu^{*} \sigma_{3} M_{2} \int_{0}^{1} h_{2}(s)\left(\alpha_{2}(s)+p_{2}(s)\right) d s+\lambda^{*} \sigma_{4} M_{1} \int_{0}^{1} h_{1}(s)\left(\alpha_{1}(s)+p_{1}(s)\right) d s \\
\leq & \frac{R_{1}}{4}+\frac{R_{1}}{4}=\frac{R_{1}}{2}=\frac{\|(x, y)\|_{Y}}{2} .
\end{aligned}
$$

Therefore

$$
\|\mathcal{Q}(x, y)\|_{Y}=\left\|Q_{1}(x, y)\right\|+\left\|Q_{2}(x, y)\right\| \leq\|(x, y)\|_{Y}, \quad \forall(x, y) \in P \cap \partial \Omega_{1} .
$$

On the other hand, we choose a constant $L>0$ such that

$$
\begin{aligned}
& \lambda L \varrho_{1} \gamma_{1} c^{2(\alpha-1)} \int_{c}^{1-c} h_{1}(s) d s \geq 4, \lambda L \varrho_{4} \gamma_{2} c^{2(\beta-1)} \int_{c}^{1-c} h_{1}(s) d s \geq 4, \\
& \mu L \varrho_{2} \gamma_{1} c^{2(\alpha-1)} \int_{c}^{1-c} h_{2}(s) d s \geq 4, \mu L \varrho_{3} \gamma_{2} c^{2(\beta-1)} \int_{c}^{1-c} h_{2}(s) d s \geq 4 .
\end{aligned}
$$

From (H5), we deduce that there exists a constant $M_{0}>0$ such that

$$
f(t, u, v) \geq L(u+v) \quad \text { or } \quad g(t, u, v) \geq L(u+v), \quad \forall t \in[c, 1-c], u, v \geq 0, u+v \geq M_{0} .
$$

Now we define

$$
\begin{aligned}
R_{2}= & \max \left\{2 R_{1}, \frac{4 M_{0}}{\gamma_{1} c^{\alpha-1}}, \frac{4 M_{0}}{\gamma_{2} c^{\beta-1}}, \frac{4}{\gamma_{1}} \int_{0}^{1}\left(\delta_{1} p_{1}(s)+\delta_{2} p_{2}(s)\right) d s,\right. \\
& \left.\frac{4}{\gamma_{2}} \int_{0}^{1}\left(\delta_{3} p_{2}(s)+\delta_{4} p_{1}(s)\right) d s\right\}>0,
\end{aligned}
$$

and let $\Omega_{2}=\left\{(x, y) \in P,\|(x, y)\|_{Y}<R_{2}\right\}$.

We suppose that $f_{\infty}=\infty$, that is, $f(t, u, v) \geq L(u+v)$ for all $t \in[c, 1-c]$ and $u, v \geq 0$, $u+v \geq M_{0}$. Then, for any $(x, y) \in P \cap \partial \Omega_{2}$, we have $\|(x, y)\|_{Y}=R_{2}$ or $\|x\|+\|y\|=R_{2}$. We deduce that $\|x\| \geq \frac{R_{2}}{2}$ or $\|y\| \geq \frac{R_{2}}{2}$. 
We suppose that $\|x\| \geq \frac{R_{2}}{2}$. Then, for any $(x, y) \in P \cap \partial \Omega_{2}$, we obtain

$$
\begin{aligned}
x(t)-q_{1}(t) & =x(t)-\lambda \int_{0}^{1} G_{1}(t, s) p_{1}(s) d s-\mu \int_{0}^{1} G_{2}(t, s) p_{2}(s) d s \\
& \geq x(t)-t^{\alpha-1}\left(\delta_{1} \int_{0}^{1} p_{1}(s) d s+\delta_{2} \int_{0}^{1} p_{2}(s) d s\right) \\
& \geq x(t)-\frac{x(t)}{\gamma_{1}\|x\|} \int_{0}^{1}\left(\delta_{1} p_{1}(s)+\delta_{2} p_{2}(s)\right) d s \\
& =x(t)\left[1-\frac{1}{\gamma_{1}\|x\|} \int_{0}^{1}\left(\delta_{1} p_{1}(s)+\delta_{2} p_{2}(s)\right) d s\right] \\
& \geq x(t)\left[1-\frac{2}{\gamma_{1} R_{2}} \int_{0}^{1}\left(\delta_{1} p_{1}(s)+\delta_{2} p_{2}(s)\right) d s\right] \geq \frac{1}{2} x(t) \geq 0, \quad \forall t \in[0,1] .
\end{aligned}
$$

Therefore, we conclude

$$
\begin{aligned}
{\left[x(t)-q_{1}(t)\right]^{*} } & =x(t)-q_{1}(t) \geq \frac{1}{2} x(t) \geq \frac{1}{2} \gamma_{1} t^{\alpha-1}\|x\| \\
& \geq \frac{1}{4} \gamma_{1} t^{\alpha-1} R_{2} \geq \frac{1}{4} \gamma_{1} c^{\alpha-1} R_{2} \geq M_{0}, \quad \forall t \in[c, 1-c] .
\end{aligned}
$$

Hence

$$
\left[x(t)-q_{1}(t)\right]^{*}+\left[y(t)-q_{2}(t)\right]^{*} \geq\left[x(t)-q_{1}(t)\right]^{*}=x(t)-q_{1}(t) \geq M_{0}, \quad \forall t \in[c, 1-c] .
$$

Then, for any $(x, y) \in P \cap \partial \Omega_{2}$ and $t \in[c, 1-c]$, by (12) and (13), we deduce

$$
\begin{aligned}
f\left(t,\left[x(t)-q_{1}(t)\right]^{*},\left[y(t)-q_{2}(t)\right]^{*}\right) & \geq L\left(\left[x(t)-q_{1}(t)\right]^{*}+\left[y(t)-q_{2}(t)\right]^{*}\right) \\
& \geq L\left[x(t)-q_{1}(t)\right]^{*} \geq \frac{L}{2} x(t), \quad \forall t \in[c, 1-c] .
\end{aligned}
$$

It follows that, for any $(x, y) \in P \cap \partial \Omega_{2}, t \in[c, 1-c]$, we obtain

$$
\begin{aligned}
Q_{1}(x, y)(t) & \geq \lambda \int_{0}^{1} G_{1}(t, s)\left(f\left(s,\left[x(s)-q_{1}(s)\right]^{*},\left[y(s)-q_{2}(s)\right]^{*}\right)+p_{1}(s)\right) d s \\
& \geq \lambda \int_{c}^{1-c} G_{1}(t, s)\left(f\left(s,\left[x(s)-q_{1}(s)\right]^{*},\left[y(s)-q_{2}(s)\right]^{*}\right)+p_{1}(s)\right) d s \\
& \geq \lambda \int_{c}^{1-c} G_{1}(t, s) L\left(\left[x(s)-q_{1}(s)\right]^{*}\right) d s \geq \lambda \int_{c}^{1-c} G_{1}(t, s) \frac{1}{4} L \gamma_{1} c^{\alpha-1} R_{2} d s \\
& \geq \lambda \int_{c}^{1-c} \varrho_{1} t^{\alpha-1} h_{1}(s) \frac{1}{4} L \gamma_{1} c^{\alpha-1} R_{2} d s \\
& \geq \lambda c^{2(\alpha-1)} \frac{1}{4} \varrho_{1} L \gamma_{1} R_{2} \int_{c}^{1-c} h_{1}(s) d s \geq R_{2} .
\end{aligned}
$$

Then $\left\|Q_{1}(x, y)\right\| \geq\|(x, y)\|_{Y}$ and

$$
\|\mathcal{Q}(x, y)\|_{Y} \geq\|(x, y)\|_{Y}, \quad \forall(x, y) \in P \cap \partial \Omega_{2} .
$$


If $\|y\| \geq \frac{R_{2}}{2}$, then by a similar approach, we obtain again relation (14).

We suppose now that $g_{\infty}=\infty$, that is, $g(t, u, v) \geq L(u+v)$, for all $t \in[c, 1-c]$ and $u, v \geq 0$, $u+v \geq M_{0}$. Then, for any $(x, y) \in P \cap \partial \Omega_{2}$, we have $\|(x, y)\|_{Y}=R_{2}$. Hence $\|x\| \geq \frac{R_{2}}{2}$ or $\|y\| \geq \frac{R_{2}}{2}$.

If $\|x\| \geq \frac{R_{2}}{2}$, then for any $(x, y) \in P \cap \partial \Omega_{2}$ we deduce in a similar manner as above that $x(t)-q_{1}(t) \geq \frac{1}{2} x(t)$ for all $t \in[0,1]$ and

$$
\begin{aligned}
Q_{1}(x, y)(t) & \geq \mu \int_{0}^{1} G_{2}(t, s)\left(g\left(s,\left[x(s)-q_{1}(s)\right]^{*},\left[y(s)-q_{2}(s)\right]^{*}\right)+p_{2}(s)\right) d s \\
& \geq \mu \int_{c}^{1-c} G_{2}(t, s)\left(g\left(s,\left[x(s)-q_{1}(s)\right]^{*},\left[y(s)-q_{2}(s)\right]^{*}\right)+p_{2}(s)\right) d s \\
& \geq \mu \int_{c}^{1-c} G_{2}(t, s) L\left(\left[x(s)-q_{1}(s)\right]^{*}\right) d s \geq \mu \int_{c}^{1-c} G_{2}(t, s) \frac{1}{4} L \gamma_{1} c^{\alpha-1} R_{2} d s \\
& \geq \mu \int_{c}^{1-c} \varrho_{2} t^{\alpha-1} h_{2}(s) \frac{1}{4} L \gamma_{1} c^{\alpha-1} R_{2} d s \geq \mu c^{2(\alpha-1)} \frac{1}{4} \varrho_{2} L \gamma_{1} R_{2} \int_{c}^{1-c} h_{2}(s) d s \\
& \geq R_{2}, \quad \forall t \in[c, 1-c] .
\end{aligned}
$$

Hence we obtain relation (14).

If $\|y\| \geq \frac{R_{2}}{2}$, then in a similar way as above, we deduce again relation (14).

Therefore, by Theorem 2.2, relations (11) and (14), we conclude that $\mathcal{Q}$ has a fixed point $\left(x_{1}, y_{1}\right) \in P \cap\left(\bar{\Omega}_{2} \backslash \Omega_{1}\right)$, that is, $R_{1} \leq\left\|\left(x_{1}, y_{1}\right)\right\|_{Y} \leq R_{2}$. Since $\left\|\left(x_{1}, y_{1}\right)\right\|_{Y} \geq R_{1}$, then $\left\|x_{1}\right\| \geq \frac{R_{1}}{2}$ or $\left\|y_{1}\right\| \geq \frac{R_{1}}{2}$.

We suppose first that $\left\|x_{1}\right\| \geq \frac{R_{1}}{2}$. Then we deduce

$$
\begin{aligned}
x_{1}(t)-q_{1}(t) & =x_{1}(t)-\lambda \int_{0}^{1} G_{1}(t, s) p_{1}(s) d s-\mu \int_{0}^{1} G_{2}(t, s) p_{2}(s) d s \\
& \geq x_{1}(t)-t^{\alpha-1}\left(\delta_{1} \int_{0}^{1} p_{1}(s) d s+\delta_{2} \int_{0}^{1} p_{2}(s) d s\right) \\
& \geq x_{1}(t)-\frac{x_{1}(t)}{\gamma_{1}\left\|x_{1}\right\|} \int_{0}^{1}\left(\delta_{1} p_{1}(s)+\delta_{2} p_{2}(s)\right) d s \\
& \geq\left[1-\frac{2}{\gamma_{1} R_{1}} \int_{0}^{1}\left(\delta_{1} p_{1}(s)+\delta_{2} p_{2}(s)\right) d s\right] x_{1}(t) \\
& \geq\left[1-\frac{2}{\gamma_{1} R_{1}} \int_{0}^{1}\left(\delta_{1} p_{1}(s)+\delta_{2} p_{2}(s)\right) d s\right] \gamma_{1} t^{\alpha-1}\left\|x_{1}\right\| \\
& \geq \frac{R_{1}}{2}\left[1-\frac{2}{\gamma_{1} R_{1}} \int_{0}^{1}\left(\delta_{1} p_{1}(s)+\delta_{2} p_{2}(s)\right) d s\right] \gamma_{1} t^{\alpha-1} \\
& =\Lambda_{1} t^{\alpha-1}, \quad \forall t \in[0,1],
\end{aligned}
$$

and so $x_{1}(t) \geq q_{1}(t)+\Lambda_{1} t^{\alpha-1}$ for all $t \in[0,1]$, where $\Lambda_{1}=\frac{\gamma_{1} R_{1}}{2}-\int_{0}^{1}\left(\delta_{1} p_{1}(s)+\delta_{2} p_{2}(s)\right) d s>0$.

Then $y_{1}(1)=\int_{0}^{1} x_{1}(s) d K(s) \geq \Lambda_{1} \int_{0}^{1} s^{\alpha-1} d K(s)>0$ and

$$
\begin{aligned}
\left\|y_{1}\right\| & \geq y_{1}(1)=\int_{0}^{1} x_{1}(s) d K(s) \geq \int_{0}^{1} \gamma_{1} s^{\alpha-1}\left\|x_{1}\right\| d K(s) \\
& \geq \frac{\gamma_{1} R_{1}}{2} \int_{0}^{1} s^{\alpha-1} d K(s)>0 .
\end{aligned}
$$


Therefore, we obtain

$$
\begin{aligned}
y_{1}(t)-q_{2}(t)= & y_{1}(t)-\mu \int_{0}^{1} G_{3}(t, s) p_{2}(s) d s-\lambda \int_{0}^{1} G_{4}(t, s) p_{1}(s) d s \\
\geq & y_{1}(t)-t^{\beta-1} \int_{0}^{1}\left(\delta_{3} p_{2}(s)+\delta_{4} p_{1}(s)\right) d s \\
\geq & y_{1}(t)-\frac{y_{1}(t)}{\gamma_{2}\left\|y_{1}\right\|} \int_{0}^{1}\left(\delta_{3} p_{2}(s)+\delta_{4} p_{1}(s)\right) d s \\
\geq & y_{1}(t)\left[1-\frac{2}{\gamma_{1} \gamma_{2} R_{1}}\left(\int_{0}^{1} s^{\alpha-1} d K(s)\right)^{-1} \int_{0}^{1}\left(\delta_{3} p_{2}(s)+\delta_{4} p_{1}(s)\right) d s\right] \\
\geq & \gamma_{2} t^{\beta-1}\left\|y_{1}\right\|\left[1-\frac{2}{\gamma_{1} \gamma_{2} R_{1}}\left(\int_{0}^{1} s^{\alpha-1} d K(s)\right)^{-1} \int_{0}^{1}\left(\delta_{3} p_{2}(s)+\delta_{4} p_{1}(s)\right) d s\right] \\
\geq & \frac{\gamma_{1} \gamma_{2} R_{1}}{2} t^{\beta-1} \int_{0}^{1} s^{\alpha-1} d K(s) \\
& \times\left[1-\frac{2}{\gamma_{1} \gamma_{2} R_{1}}\left(\int_{0}^{1} s^{\alpha-1} d K(s)\right)^{-1} \int_{0}^{1}\left(\delta_{3} p_{2}(s)+\delta_{4} p_{1}(s)\right) d s\right] \\
= & \Lambda_{2} t^{\beta-1}, \forall t \in[0,1],
\end{aligned}
$$

where $\Lambda_{2}=\frac{\gamma_{1} \gamma_{2} R_{1}}{2} \int_{0}^{1} s^{\alpha-1} d K(s)-\int_{0}^{1}\left(\delta_{3} p_{2}(s)+\delta_{4} p_{1}(s)\right) d s>0$.

Hence $y_{1}(t) \geq q_{2}(t)+\Lambda_{2} t^{\beta-1}$ for all $t \in[0,1]$.

If $\left\|y_{1}\right\| \geq \frac{R_{1}}{2}$, then by a similar approach, we deduce that $y_{1}(t) \geq q_{2}(t)+\Lambda_{3} t^{\beta-1}$ and $x_{1}(t) \geq q_{1}(t)+\Lambda_{4} t^{\alpha-1}$ for all $t \in[0,1]$, where $\Lambda_{3}=\frac{\gamma_{2} R_{1}}{2}-\int_{0}^{1}\left(\delta_{3} p_{2}(s)+\delta_{4} p_{1}(s)\right) d s>0$ and $\Lambda_{4}=\frac{\gamma_{1} \gamma_{2} R_{1}}{2} \int_{0}^{1} s^{\beta-1} d H(s)-\int_{0}^{1}\left(\delta_{1} p_{1}(s)+\delta_{2} p_{2}(s)\right) d s>0$

Let $u_{1}(t)=x_{1}(t)-q_{1}(t)$ and $v_{1}(t)=y_{1}(t)-q_{2}(t)$ for all $t \in[0,1]$. Then $\left(u_{1}, v_{1}\right)$ is a positive solution of (S)-(BC) with $u_{1}(t) \geq \Lambda_{5} t^{\alpha-1}$ and $v_{1}(t) \geq \Lambda_{6} t^{\beta-1}$ for all $t \in[0,1]$, where $\Lambda_{5}=$ $\min \left\{\Lambda_{1}, \Lambda_{4}\right\}$ and $\Lambda_{6}=\min \left\{\Lambda_{2}, \Lambda_{3}\right\}$. This completes the proof of Theorem 3.2.

Theorem 3.3 Assume that (H1), (H3), (H5), and

(H4') The functions $f, g \in C([0,1] \times[0, \infty) \times[0, \infty),(-\infty,+\infty))$ and there exist functions $p_{1}, p_{2}, \alpha_{1}, \alpha_{2} \in C([0,1],[0, \infty)), \beta_{1}, \beta_{2} \in C([0,1] \times[0, \infty) \times[0, \infty),[0, \infty))$ such that

$$
-p_{1}(t) \leq f(t, u, v) \leq \alpha_{1}(t) \beta_{1}(t, u, v), \quad-p_{2}(t) \leq g(t, u, v) \leq \alpha_{2}(t) \beta_{2}(t, u, v),
$$

for all $t \in[0,1], u, v \in[0, \infty)$, with $\int_{0}^{1} p_{i}(s) d s>0, i=1,2$,

hold. Then the boundary value problem (S)-(BC) has at least two positive solutions for $\lambda>0$ and $\mu>0$ sufficiently small.

Proof Because assumption (H4') implies assumptions (H2) and (H4), we can apply Theorems 3.1 and 3.2. Therefore, we deduce that, for $0<\lambda \leq \min \left\{\lambda_{0}, \lambda^{*}\right\}$ and $0<\mu \leq$ $\min \left\{\mu_{0}, \mu^{*}\right\}$, problem $(\mathrm{S})-(\mathrm{BC})$ has at least two positive solutions $\left(u_{0}, v_{0}\right)$ and $\left(u_{1}, v_{1}\right)$ with $\left\|\left(u_{0}+q_{1}, v_{0}+q_{2}\right)\right\|_{Y} \leq 1$ and $\left\|\left(u_{1}+q_{1}, v_{1}+q_{2}\right)\right\|_{Y}>1$.

Theorem 3.4 Assume that $\lambda=\mu$, and (H1), (H4), and (H6) hold. In addition if 
(H7) there exists $c \in(0,1 / 2)$ such that

$$
f_{\infty}^{i}=\liminf _{\substack{u+v \rightarrow \infty \\ u, v \geq 0}} \min _{t \in[c, 1-c]} f(t, u, v)>L_{0} \quad \text { or } \quad g_{\infty}^{i}=\liminf _{\substack{u+v \rightarrow \infty \\ u, v \geq 0}} \min _{\substack{t \in[c, 1-c]\\}} g(t, u, v)>L_{0}
$$

where

$$
\begin{aligned}
L_{0}= & \max \left\{\frac{4}{\gamma_{1}} \int_{0}^{1}\left(\delta_{1} p_{1}(s)+\delta_{2} p_{2}(s)\right) d s, \frac{4}{\gamma_{2}} \int_{0}^{1}\left(\delta_{3} p_{2}(s)+\delta_{4} p_{1}(s)\right) d s,\right. \\
& \frac{4}{\gamma_{1} \gamma_{2}}\left(\int_{0}^{1} s^{\alpha-1} d K(s)\right)^{-1} \int_{0}^{1}\left(\delta_{3} p_{2}(s)+\delta_{4} p_{1}(s)\right) d s \\
& \left.\frac{4}{\gamma_{1} \gamma_{2}}\left(\int_{0}^{1} s^{\beta-1} d H(s)\right)^{-1} \int_{0}^{1}\left(\delta_{1} p_{1}(s)+\delta_{2} p_{2}(s)\right) d s\right\} \\
& \times\left(\min \left\{c^{\alpha-1} \varrho_{1} \int_{c}^{1-c} h_{1}(s) d s, c^{\alpha-1} \varrho_{2} \int_{c}^{1-c} h_{2}(s) d s\right\}\right)^{-1}
\end{aligned}
$$

then there exists $\lambda_{*}>0$ such that for any $\lambda \geq \lambda_{*}$ problem $(\mathrm{S})-(\mathrm{BC})($ with $\lambda=\mu)$ has at least one positive solution.

Proof By (H7) we conclude that there exists $M_{3}>0$ such that

$$
f(t, u, v) \geq L_{0} \quad \text { or } \quad g(t, u, v) \geq L_{0}, \quad \forall t \in[c, 1-c], u, v \geq 0, u+v \geq M_{3}
$$

We define

$$
\lambda_{*}=\max \left\{\frac{M_{3}}{c^{\alpha-1}}\left(\int_{0}^{1}\left(\delta_{1} p_{1}(s)+\delta_{2} p_{2}(s)\right) d s\right)^{-1}, \frac{M_{3}}{c^{\beta-1}}\left(\int_{0}^{1}\left(\delta_{3} p_{2}(s)+\delta_{4} p_{1}(s)\right) d s\right)^{-1}\right\} .
$$

We assume now $\lambda \geq \lambda_{*}$. Let

$$
\begin{aligned}
R_{3}= & \max \left\{\frac{4 \lambda}{\gamma_{1}} \int_{0}^{1}\left(\delta_{1} p_{1}(s)+\delta_{2} p_{2}(s)\right) d s, \frac{4 \lambda}{\gamma_{2}} \int_{0}^{1}\left(\delta_{3} p_{2}(s)+\delta_{4} p_{1}(s)\right) d s,\right. \\
& \frac{4 \lambda}{\gamma_{1} \gamma_{2}}\left(\int_{0}^{1} s^{\alpha-1} d K(s)\right)^{-1} \int_{0}^{1}\left(\delta_{3} p_{2}(s)+\delta_{4} p_{1}(s)\right) d s, \\
& \left.\frac{4 \lambda}{\gamma_{1} \gamma_{2}}\left(\int_{0}^{1} s^{\beta-1} d H(s)\right)^{-1} \int_{0}^{1}\left(\delta_{1} p_{1}(s)+\delta_{2} p_{2}(s)\right) d s\right\},
\end{aligned}
$$

and $\Omega_{3}=\left\{(x, y) \in P,\|(x, y)\|_{Y}<R_{3}\right\}$.

We suppose first that $f_{\infty}^{i}>L_{0}$, that is, $f(t, u, v) \geq L_{0}$ for all $t \in[c, 1-c]$ and $u, v \geq 0$, $u+v \geq M_{3}$. Let $(x, y) \in P \cap \partial \Omega_{3}$. Then $\|(x, y)\|_{Y}=R_{3}$, so $\|x\| \geq R_{3} / 2$ or $\|y\| \geq R_{3} / 2$. We assume that $\|x\| \geq R_{3} / 2$. Then for all $t \in[0,1]$ we deduce

$$
\begin{aligned}
x(t)-q_{1}(t) & \geq \gamma_{1} t^{\alpha-1}\|x\|-\lambda t^{\alpha-1} \delta_{1} \int_{0}^{1} p_{1}(s) d s-\lambda t^{\alpha-1} \delta_{2} \int_{0}^{1} p_{2}(s) d s \\
& \geq t^{\alpha-1}\left[\frac{\gamma_{1} R_{3}}{2}-\lambda \int_{0}^{1}\left(\delta_{1} p_{1}(s)+\delta_{2} p_{2}(s)\right) d s\right] \\
& \geq t^{\alpha-1}\left[2 \lambda \int_{0}^{1}\left(\delta_{1} p_{1}(s)+\delta_{2} p_{2}(s)\right) d s-\lambda \int_{0}^{1}\left(\delta_{1} p_{1}(s)+\delta_{2} p_{2}(s)\right) d s\right]
\end{aligned}
$$




$$
\begin{aligned}
& =t^{\alpha-1} \lambda \int_{0}^{1}\left(\delta_{1} p_{1}(s)+\delta_{2} p_{2}(s)\right) d s \\
& \geq t^{\alpha-1} \lambda_{*} \int_{0}^{1}\left(\delta_{1} p_{1}(s)+\delta_{2} p_{2}(s)\right) d s \geq \frac{M_{3}}{c^{\alpha-1}} t^{\alpha-1} \geq 0 .
\end{aligned}
$$

Therefore, for any $(x, y) \in P \cap \partial \Omega_{3}$ and $t \in[c, 1-c]$, we have

$$
\left[x(t)-q_{1}(t)\right]^{*}+\left[y(t)-q_{2}(t)\right]^{*} \geq\left[x(t)-q_{1}(t)\right]^{*}=x(t)-q_{1}(t) \geq \frac{M_{3}}{c^{\alpha-1}} t^{\alpha-1} \geq M_{3} .
$$

Hence, for any $(x, y) \in P \cap \partial \Omega_{3}$ and $t \in[c, 1-c]$, we conclude

$$
\begin{aligned}
Q_{1}(x, y)(t) & \geq \lambda \int_{0}^{1} G_{1}(t, s)\left[f\left(s,\left[x(s)-q_{1}(s)\right]^{*},\left[y(s)-q_{2}(s)\right]^{*}\right)+p_{1}(s)\right] d s \\
& \geq \lambda \varrho_{1} t^{\alpha-1} \int_{c}^{1-c} h_{1}(s) f\left(s,\left[x(s)-q_{1}(s)\right]^{*},\left[y(s)-q_{2}(s)\right]^{*}\right) d s \\
& \geq \lambda L_{0} \varrho_{1} t^{\alpha-1} \int_{c}^{1-c} h_{1}(s) d s \geq \lambda L_{0} \varrho_{1} c^{\alpha-1} \int_{c}^{1-c} h_{1}(s) d s \geq R_{3}=\|(x, y)\|_{Y}
\end{aligned}
$$

Therefore we obtain $\left\|Q_{1}(x, y)\right\| \geq R_{3}$ for all $(x, y) \in P \cap \partial \Omega_{3}$, and so

$$
\|\mathcal{Q}(x, y)\|_{Y} \geq R_{3}=\|(x, y)\|_{Y}, \quad \forall(x, y) \in P \cap \partial \Omega_{3} .
$$

If $\|y\| \geq R_{3} / 2$, then by a similar approach we deduce again relation (16).

We suppose now that $g_{\infty}^{i}>L_{0}$, that is, $g(t, u, v) \geq L_{0}$ for all $t \in[c, 1-c]$ and $u, v \geq 0$, $u+v \geq M_{3}$. Let $(x, y) \in P \cap \partial \Omega_{3}$. Then $\|(x, y)\|_{Y}=R_{3}$, so $\|x\| \geq R_{3} / 2$ or $\|y\| \geq R_{3} / 2$. If $\|x\| \geq$ $R_{3} / 2$, then we obtain in a similar manner as in the first case above $\left(f_{\infty}^{i}>L_{0}\right)$ that $x(t)-$ $q_{1}(t) \geq \frac{M_{3}}{c^{\alpha-1}} t^{\alpha-1} \geq 0$ for all $t \in[0,1]$.

Therefore, for any $(x, y) \in P \cap \partial \Omega_{3}$ and $t \in[c, 1-c]$, we deduce inequalities (15).

Hence, for any $(x, y) \in P \cap \partial \Omega_{3}$ and $t \in[c, 1-c]$, we conclude

$$
\begin{aligned}
Q_{1}(x, y)(t) & \geq \lambda \int_{0}^{1} G_{2}(t, s)\left[g\left(s,\left[x(s)-q_{1}(s)\right]^{*},\left[y(s)-q_{2}(s)\right]^{*}\right)+p_{2}(s)\right] d s \\
& \geq \lambda \varrho_{2} t^{\alpha-1} \int_{c}^{1-c} h_{2}(s) g\left(s,\left[x(s)-q_{1}(s)\right]^{*},\left[y(s)-q_{2}(s)\right]^{*}\right) d s \\
& \geq \lambda L_{0} \varrho_{2} t^{\alpha-1} \int_{c}^{1-c} h_{2}(s) d s \geq \lambda L_{0} \varrho_{2} c^{\alpha-1} \int_{c}^{1-c} h_{2}(s) d s \geq R_{3}=\|(x, y)\|_{Y} .
\end{aligned}
$$

Therefore we obtain $\left\|Q_{1}(x, y)\right\| \geq R_{3}$, and so $\|\mathcal{Q}(x, y)\|_{Y} \geq R_{3}=\|(x, y)\|_{Y}$ for all $(x, y) \in$ $P \cap \partial \Omega_{3}$, that is, we have relation (16).

By a similar approach we obtain relation (16) if $\|y\| \geq R_{3} / 2$.

On the other hand, we consider the positive number

$$
\begin{aligned}
\varepsilon= & \min \left\{\frac{1}{8 \lambda \sigma_{1}}\left(\int_{0}^{1} h_{1}(s) \alpha_{1}(s) d s\right)^{-1}, \frac{1}{8 \lambda \sigma_{2}}\left(\int_{0}^{1} h_{2}(s) \alpha_{2}(s) d s\right)^{-1},\right. \\
& \left.\frac{1}{8 \lambda \sigma_{3}}\left(\int_{0}^{1} h_{2}(s) \alpha_{2}(s) d s\right)^{-1}, \frac{1}{8 \lambda \sigma_{4}}\left(\int_{0}^{1} h_{1}(s) \alpha_{1}(s) d s\right)^{-1}\right\} .
\end{aligned}
$$


Then by (H6) we deduce that there exists $M_{4}>0$ such that

$$
\beta_{i}(t, u, v) \leq \varepsilon(u+v), \quad \forall t \in[0,1], u, v \geq 0, u+v \geq M_{4}, i=1,2
$$

Therefore we obtain

$$
\beta_{i}(t, u, v) \leq M_{5}+\varepsilon(u+v), \quad \forall t \in[0,1], u, v \geq 0, i=1,2,
$$

where $M_{5}=\max _{i=1,2}\left\{\max _{t \in[0,1], u, v \geq 0, u+v \leq M_{4}} \beta_{i}(t, u, v)\right\}$.

We define now

$$
\begin{aligned}
R_{4}= & \max \left\{2 R_{3}, 8 \lambda \sigma_{1} \max \left\{M_{5}, 1\right\} \int_{0}^{1} h_{1}(s)\left(\alpha_{1}(s)+p_{1}(s)\right) d s,\right. \\
& 8 \lambda \sigma_{2} \max \left\{M_{5}, 1\right\} \int_{0}^{1} h_{2}(s)\left(\alpha_{2}(s)+p_{2}(s)\right) d s, \\
& 8 \lambda \sigma_{3} \max \left\{M_{5}, 1\right\} \int_{0}^{1} h_{2}(s)\left(\alpha_{2}(s)+p_{2}(s)\right) d s, \\
& \left.8 \lambda \sigma_{4} \max \left\{M_{5}, 1\right\} \int_{0}^{1} h_{1}(s)\left(\alpha_{1}(s)+p_{1}(s)\right) d s\right\},
\end{aligned}
$$

and let $\Omega_{4}=\left\{(x, y) \in P,\|(x, y)\|_{Y}<R_{4}\right\}$.

For any $(x, y) \in P \cap \partial \Omega_{4}$, we have

$$
\begin{aligned}
Q_{1}(x, y)(t) \leq & \lambda \int_{0}^{1} \sigma_{1} h_{1}(s)\left[\alpha_{1}(s) \beta_{1}\left(s,\left[x(s)-q_{1}(s)\right]^{*},\left[y(s)-q_{2}(s)\right]^{*}\right)+p_{1}(s)\right] d s \\
& +\lambda \int_{0}^{1} \sigma_{2} h_{2}(s)\left[\alpha_{2}(s) \beta_{2}\left(s,\left[x(s)-q_{1}(s)\right]^{*},\left[y(s)-q_{2}(s)\right]^{*}\right)+p_{2}(s)\right] d s \\
\leq & \lambda \sigma_{1} \int_{0}^{1} h_{1}(s)\left[\alpha_{1}(s)\left(M_{5}+\varepsilon\left(\left[x(s)-q_{1}(s)\right]^{*}+\left[y(s)-q_{2}(s)\right]^{*}\right)\right)+p_{1}(s)\right] d s \\
& +\lambda \sigma_{2} \int_{0}^{1} h_{2}(s)\left[\alpha_{2}(s)\left(M_{5}+\varepsilon\left(\left[x(s)-q_{1}(s)\right]^{*}+\left[y(s)-q_{2}(s)\right]^{*}\right)\right)+p_{2}(s)\right] d s \\
\leq & \lambda \sigma_{1} \max \left\{M_{5}, 1\right\} \int_{0}^{1} h_{1}(s)\left(\alpha_{1}(s)+p_{1}(s)\right) d s+\lambda \sigma_{1} \varepsilon R_{4} \int_{0}^{1} h_{1}(s) \alpha_{1}(s) d s \\
& +\lambda \sigma_{2} \max \left\{M_{5}, 1\right\} \int_{0}^{1} h_{2}(s)\left(\alpha_{2}(s)+p_{2}(s)\right) d s+\lambda \sigma_{2} \varepsilon R_{4} \int_{0}^{1} h_{2}(s) \alpha_{2}(s) d s \\
\leq & \frac{R_{4}}{8}+\frac{R_{4}}{8}+\frac{R_{4}}{8}+\frac{R_{4}}{8}=\frac{R_{4}}{2}=\frac{\|(x, y)\|_{Y}}{2}, \quad \forall t \in[0,1],
\end{aligned}
$$

and so $\left\|Q_{1}(x, y)\right\| \leq \frac{\|(x, y)\|_{Y}}{2}$ for all $(x, y) \in P \cap \partial \Omega_{4}$.

In a similar way we obtain $Q_{2}(x, y)(t) \leq \frac{\|(x, y)\|_{Y}}{2}$ for all $t \in[0,1]$, and so $\left\|Q_{2}(x, y)\right\| \leq \frac{\|(x, y)\|_{Y}}{2}$ for all $(x, y) \in P \cap \partial \Omega_{4}$.

Therefore, we deduce

$$
\|\mathcal{Q}(x, y)\|_{Y} \leq\|(x, y)\|_{Y}, \quad \forall(x, y) \in P \cap \partial \Omega_{4}
$$

By Theorem 2.2, (16), and (17), we conclude that $\mathcal{Q}$ has a fixed point $\left(x_{1}, y_{1}\right) \in P \cap\left(\bar{\Omega}_{4} \backslash\right.$ $\left.\Omega_{3}\right)$. Since $\left\|\left(x_{1}, y_{1}\right)\right\| \geq R_{3}$ then $\left\|x_{1}\right\| \geq R_{3} / 2$ or $\left\|y_{1}\right\| \geq R_{3} / 2$. 
We suppose that $\left\|x_{1}\right\| \geq R_{3} / 2$. Then $x_{1}(t)-q_{1}(t) \geq \frac{M_{3}}{c^{\alpha-1}} t^{\alpha-1}$ for all $t \in[0,1]$. Besides

$$
y_{1}(1)=\int_{0}^{1} x_{1}(s) d K(s) \geq \gamma_{1}\left\|x_{1}\right\| \int_{0}^{1} s^{\alpha-1} d K(s) \geq \frac{\gamma_{1} R_{3}}{2} \int_{0}^{1} s^{\alpha-1} d K(s)>0,
$$

and then

$$
\left\|y_{1}\right\| \geq y_{1}(1)=\int_{0}^{1} x_{1}(s) d K(s) \geq \frac{\gamma_{1} R_{3}}{2} \int_{0}^{1} s^{\alpha-1} d K(s)>0 .
$$

Therefore, we deduce that, for all $t \in[0,1]$,

$$
\begin{aligned}
y_{1}(t)-q_{2}(t) & \geq y_{1}(t)-\lambda \delta_{3} \int_{0}^{1} t^{\beta-1} p_{2}(s) d s-\lambda \delta_{4} \int_{0}^{1} t^{\beta-1} p_{1}(s) d s \\
& \geq \gamma_{2} t^{\beta-1}\left\|y_{1}\right\|-\lambda t^{\beta-1} \int_{0}^{1}\left(\delta_{3} p_{2}(s)+\delta_{4} p_{1}(s)\right) d s \\
& \geq \frac{\gamma_{1} \gamma_{2} R_{3}}{2} t^{\beta-1} \int_{0}^{1} s^{\alpha-1} d K(s)-\lambda t^{\beta-1} \int_{0}^{1}\left(\delta_{3} p_{2}(s)+\delta_{4} p_{1}(s)\right) d s \\
& \geq \lambda t^{\beta-1} \int_{0}^{1}\left(\delta_{3} p_{2}(s)+\delta_{4} p_{1}(s)\right) d s \\
& \geq \lambda_{*} t^{\beta-1} \int_{0}^{1}\left(\delta_{3} p_{2}(s)+\delta_{4} p_{1}(s)\right) d s \geq \frac{M_{3}}{c^{\beta-1}} t^{\beta-1} .
\end{aligned}
$$

If $\left\|y_{1}\right\| \geq R_{3} / 2$, then by a similar approach we conclude again that $x_{1}(t)-q_{1}(t) \geq \frac{M_{3}}{c^{\alpha-1}} t^{\alpha-1}$ and $y_{1}(t)-q_{2}(t) \geq \frac{M_{3}}{c^{\beta-1}} t^{\beta-1}$ for all $t \in[0,1]$.

Let $u_{1}(t)=x_{1}(t)-q_{1}(t)$ and $v_{1}(t)=y_{1}(t)-q_{2}(t)$ for all $t \in[0,1]$. Then $u_{1}(t) \geq \widetilde{\Lambda}_{1} t^{\alpha-1}$ and $v_{1}(t) \geq \widetilde{\Lambda}_{2} t^{\beta-1}$ for all $t \in[0,1]$, where $\widetilde{\Lambda}_{1}=\frac{M_{3}}{c^{\alpha-1}}, \widetilde{\Lambda}_{2}=\frac{M_{3}}{c^{\beta-1}}$. Hence we deduce that $\left(u_{1}, v_{1}\right)$ is a positive solution of $(\mathrm{S})-(\mathrm{BC})$, which completes the proof of Theorem 3.4.

In a similar manner as we proved Theorem 3.4, we obtain the following theorems.

Theorem 3.5 Assume that $\lambda=\mu$, and (H1), (H4), and (H6) hold. In addition if

$\left(\mathrm{H}^{\prime}\right)$ there exists $c \in(0,1 / 2)$ such that

$$
f_{\infty}^{i}=\liminf _{\substack{u+v \rightarrow \infty \\ u, v \geq 0}} \min _{t \in[c, 1-c]} f(t, u, v)>\widetilde{L}_{0} \quad \text { or } \quad g_{\infty}^{i}=\liminf _{\substack{u+v \rightarrow \infty \\ u, v \geq 0}} \min _{t \in[c, 1-c]} g(t, u, v)>\widetilde{L}_{0}
$$

where

$$
\begin{aligned}
\widetilde{L}_{0}= & \max \left\{\frac{4}{\gamma_{1}} \int_{0}^{1}\left(\delta_{1} p_{1}(s)+\delta_{2} p_{2}(s)\right) d s, \frac{4}{\gamma_{2}} \int_{0}^{1}\left(\delta_{3} p_{2}(s)+\delta_{4} p_{1}(s)\right) d s,\right. \\
& \frac{4}{\gamma_{1} \gamma_{2}}\left(\int_{0}^{1} s^{\alpha-1} d K(s)\right)^{-1} \int_{0}^{1}\left(\delta_{3} p_{2}(s)+\delta_{4} p_{1}(s)\right) d s, \\
& \left.\frac{4}{\gamma_{1} \gamma_{2}}\left(\int_{0}^{1} s^{\beta-1} d H(s)\right)^{-1} \int_{0}^{1}\left(\delta_{1} p_{1}(s)+\delta_{2} p_{2}(s)\right) d s\right\} \\
& \times\left(\min \left\{c^{\beta-1} \varrho_{3} \int_{c}^{1-c} h_{2}(s) d s, c^{\beta-1} \varrho_{4} \int_{c}^{1-c} h_{1}(s) d s\right\}\right)^{-1},
\end{aligned}
$$


then there exists $\lambda_{*}^{\prime}>0$ such that for any $\lambda \geq \lambda_{*}^{\prime}$ problem $(\mathrm{S})-(\mathrm{BC})($ with $\lambda=\mu)$ has at least one positive solution.

Theorem 3.6 Assume that $\lambda=\mu$, and (H1), (H4), and (H6) hold. In addition if (H8) there exists $c \in(0,1 / 2)$ such that

$$
\hat{f}_{\infty}=\lim _{\substack{u+v \rightarrow \infty \\ u, v \geq 0}} \min _{t \in[c, 1-c]} f(t, u, v)=\infty \quad \text { or } \quad \hat{g}_{\infty}=\lim _{\substack{u+\nu \rightarrow \infty \\ u, v \geq 0}} \min _{t \in[c, 1-c]} g(t, u, v)=\infty,
$$

then there exists $\tilde{\lambda}_{*}>0$ such that for any $\lambda \geq \tilde{\lambda}_{*}$ problem $(\mathrm{S})-(\mathrm{BC})($ with $\lambda=\mu)$ has at least one positive solution.

\section{Examples}

Let $\alpha=5 / 2(n=3), \beta=7 / 3(m=3), H(t)=t^{2}, K(t)=t^{3}$. Then $\int_{0}^{1} u(s) d K(s)=3 \int_{0}^{1} s^{2} u(s) d s$ and $\int_{0}^{1} v(s) d H(s)=2 \int_{0}^{1} s v(s) d s$.

We consider the system of fractional differential equations

$$
\left(\mathrm{S}_{0}\right) \quad \begin{cases}D_{0+}^{5 / 2} u(t)+\lambda f(t, u(t), v(t))=0, & t \in(0,1), \\ D_{0+}^{7 / 3} v(t)+\mu g(t, u(t), v(t))=0, & t \in(0,1),\end{cases}
$$

with the boundary conditions

$$
\left(\mathrm{BC}_{0}\right) \quad \begin{cases}u(0)=u^{\prime}(0)=0, & u(1)=2 \int_{0}^{1} s v(s) d s \\ v(0)=v^{\prime}(0)=0, & v(1)=3 \int_{0}^{1} s^{2} u(s) d s\end{cases}
$$

Then we obtain $\Delta=1-\left(\int_{0}^{1} s^{\alpha-1} d K(s)\right)\left(\int_{0}^{1} s^{\beta-1} d H(s)\right)=\frac{3}{5}>0, \int_{0}^{1} \tau^{\alpha-1}(1-\tau) d K(\tau)=\frac{4}{33}>$ $0, \int_{0}^{1} \tau^{\beta-1}(1-\tau) d H(\tau)=\frac{9}{65}>0$. The functions $H$ and $K$ are nondecreasing, and so assumption $(\mathrm{H} 1)$ is satisfied. Besides, we deduce

$$
\begin{aligned}
& g_{1}(t, s)=\frac{4}{3 \sqrt{\pi}} \begin{cases}t^{3 / 2}(1-s)^{3 / 2}-(t-s)^{3 / 2}, & 0 \leq s \leq t \leq 1, \\
t^{3 / 2}(1-s)^{3 / 2}, & 0 \leq t \leq s \leq 1,\end{cases} \\
& g_{2}(t, s)=\frac{1}{\Gamma(7 / 3)} \begin{cases}t^{4 / 3}(1-s)^{4 / 3}-(t-s)^{4 / 3}, & 0 \leq s \leq t \leq 1, \\
t^{4 / 3}(1-s)^{4 / 3}, & 0 \leq t \leq s \leq 1,\end{cases} \\
& G_{1}(t, s)=g_{1}(t, s)+3 t^{3 / 2} \int_{0}^{1} \tau^{2} g_{1}(\tau, s) d s, \quad G_{2}(t, s)=\frac{10}{3} t^{3 / 2} \int_{0}^{1} \tau g_{2}(\tau, s) d \tau, \\
& G_{3}(t, s)=g_{2}(t, s)+\frac{20}{9} t^{4 / 3} \int_{0}^{1} \tau g_{2}(\tau, s) d \tau, \quad G_{4}(t, s)=5 t^{4 / 3} \int_{0}^{1} \tau^{2} g_{1}(\tau, s) d \tau .
\end{aligned}
$$

We also obtain $h_{1}(s)=\frac{2}{\sqrt{\pi}} s(1-s)^{3 / 2}, h_{2}(s)=\frac{1}{\Gamma(4 / 3)} s(1-s)^{4 / 3}$,

$$
k_{1}(t)=\left\{\begin{array}{ll}
\frac{2}{3} t^{3 / 2}, & 0 \leq t \leq 1 / 2, \\
\frac{2}{3}(1-t) t^{1 / 2}, & 1 / 2 \leq t \leq 1,
\end{array} \quad k_{2}(t)= \begin{cases}\frac{3}{4} t^{4 / 3}, & 0 \leq t \leq 1 / 2, \\
\frac{3}{4}(1-t) t^{1 / 3}, & 1 / 2 \leq t \leq 1 .\end{cases}\right.
$$

In addition, we have $\sigma_{1}=2, \delta_{1}=\frac{74}{33 \sqrt{\pi}}, \varrho_{1}=\frac{8 \sqrt{2}-1}{63 \sqrt{2}}, \sigma_{2}=\frac{5}{3}, \delta_{2}=\frac{3}{13 \Gamma(4 / 3)}, \varrho_{2}=\frac{36 \sqrt[3]{2}-9}{112 \sqrt[3]{2}}, \sigma_{3}=$ $\frac{19}{9}, \delta_{3}=\frac{15}{13 \Gamma(4 / 3)}, \varrho_{3}=\frac{12 \sqrt[3]{2}-3}{56 \sqrt[3]{2}}, \sigma_{4}=\frac{5}{3}, \delta_{4}=\frac{40}{99 \sqrt{\pi}}, \varrho_{4}=\frac{40 \sqrt{2}-5}{189 \sqrt{2}}, \gamma_{1}=\frac{8 \sqrt{2}-1}{126 \sqrt{2}} \approx 0.0578801, \gamma_{2}=$ $\frac{9(12 \sqrt[3]{2}-3)}{1064 \sqrt[3]{2}} \approx 0.08136286$. 
Example 1 We consider the functions

$$
f(t, u, v)=\frac{(u+v)^{2}}{\sqrt{t(1-t)}}+\ln t, \quad g(t, u, v)=\frac{2+\sin (u+v)}{\sqrt{t(1-t)}}+\ln (1-t), \quad t \in(0,1), u, v \geq 0 .
$$

We have $p_{1}(t)=-\ln t, p_{2}(t)=-\ln (1-t), \alpha_{1}(t)=\alpha_{2}(t)=\frac{1}{\sqrt{t(1-t)}}$ for all $t \in(0,1), \beta_{1}(t, u, v)=$ $(u+v)^{2}, \beta_{2}(t, u, v)=2+\sin (u+v)$ for all $t \in[0,1], u, v \geq 0, \int_{0}^{1} p_{1}(t) d t=1, \int_{0}^{1} p_{2}(t) d t=1$, $\int_{0}^{1} \alpha_{i}(t) d t=\pi, i=1,2$. Therefore, assumption (H4) is satisfied. In addition, for $c \in(0,1 / 2)$ fixed, assumption (H5) is also satisfied $\left(f_{\infty}=\infty\right)$.

After some computations, we deduce $\int_{0}^{1}\left(\delta_{1} p_{1}(s)+\delta_{2} p_{2}(s)\right) d s \approx 1.52357852, \int_{0}^{1}\left(\delta_{3} p_{2}(s)+\right.$ $\left.\delta_{4} p_{1}(s)\right) d s \approx 1.520086, \int_{0}^{1} h_{1}(s)\left(\alpha_{1}(s)+p_{1}(s)\right) d s \approx 0.42548534, \int_{0}^{1} h_{2}(s)\left(\alpha_{2}(s)+p_{2}(s)\right) d s \approx$ 0.44092924 . We choose $R_{1}=1080$, which satisfies the condition from the beginning of the proof of Theorem 3.2. Then $M_{1}=R_{1}^{2}, M_{2}=3, \lambda^{*} \approx 2.7202 \cdot 10^{-4}$, and $\mu^{*}=1$. By Theorem 3.2, we conclude that $\left(\mathrm{S}_{0}\right)-\left(\mathrm{BC}_{0}\right)$ has at least one positive solution for any $\lambda \in\left(0, \lambda^{*}\right]$ and $\mu \in\left(0, \mu^{*}\right]$.

Example 2 We consider the functions

$$
f(t, u, v)=(u+v)^{2}+\cos u, \quad g(t, u, v)=(u+v)^{1 / 2}+\cos v, \quad t \in[0,1], u, v \geq 0 .
$$

We have $p_{1}(t)=p_{2}(t)=1$ for all $t \in[0,1]$, and then assumption (H2) is satisfied. Besides, assumption (H3) is also satisfied, because $f(t, 0,0)=1$ and $g(t, 0,0)=1$ for all $t \in[0,1]$.

Let $\delta=\frac{1}{2}<1$ and $R_{0}=1$. Then

$$
f(t, u, v) \geq \delta f(t, 0,0)=\frac{1}{2}, \quad g(t, u, v) \geq \delta g(t, 0,0)=\frac{1}{2}, \quad \forall t \in[0,1], u, v \in[0,1] .
$$

In addition,

$$
\begin{aligned}
& \bar{f}\left(R_{0}\right)=\bar{f}(1)=\max _{t \in[0,1], u, v \in[0,1]}\left\{f(t, u, v)+p_{1}(t)\right\} \approx 5.5403023, \\
& \bar{g}\left(R_{0}\right)=\bar{g}(1)=\max _{t \in[0,1], u, v \in[0,1]}\left\{g(t, u, v)+p_{2}(t)\right\} \approx 3.10479256 .
\end{aligned}
$$

We also obtain $c_{1} \approx 0.25791523, c_{2} \approx 0.23996711, c_{3} \approx 0.30395834, c_{4} \approx 0.21492936$, and then $\lambda_{0}=\max \left\{\frac{R_{0}}{8 c_{1} \bar{f}\left(R_{0}\right)}, \frac{R_{0}}{8 c_{4} \bar{f}\left(R_{0}\right)}\right\} \approx 0.10497377$ and $\mu_{0}=\max \left\{\frac{R_{0}}{8 c_{2} \bar{g}\left(R_{0}\right)}, \frac{R_{0}}{8 c_{3} \bar{g}\left(R_{0}\right)}\right\} \approx$ 0.1677744 .

By Theorem 3.1, for any $\lambda \in\left(0, \lambda_{0}\right]$ and $\mu \in\left(0, \mu_{0}\right]$, we deduce that problem $\left(\mathrm{S}_{0}\right)-\left(\mathrm{BC} \mathrm{C}_{0}\right)$ has at least one positive solution.

Because assumption $\left(\mathrm{H} 4^{\prime}\right)$ is satisfied $\left(\alpha_{1}(t)=\alpha_{2}(t)=1, \beta_{1}(t, u, v)=(u+v)^{2}+1, \beta_{2}(t, u, v)=\right.$ $(u+v)^{1 / 2}+1$ for all $\left.t \in[0,1], u, v \geq 0\right)$ and assumption (H5) is also satisfied $\left(f_{\infty}=\infty\right)$, by Theorem 3.3 we conclude that problem $\left(\mathrm{S}_{0}\right)-\left(\mathrm{BC}_{0}\right)$ has at least two positive solutions for $\lambda$ and $\mu$ sufficiently small.

Example 3 We consider $\lambda=\mu$ and the functions

$$
f(t, u, v)=\frac{(u+v)^{a}}{\sqrt[3]{t^{2}(1-t)}}-\frac{1}{\sqrt{t}}, \quad g(t, u, v)=\frac{\ln (1+u+v)}{\sqrt[3]{t(1-t)^{2}}}-\frac{1}{\sqrt{1-t}}, \quad t \in(0,1), u, v \geq 0,
$$

where $a \in(0,1)$. 
Here we have $p_{1}(t)=\frac{1}{\sqrt{t}}, p_{2}(t)=\frac{1}{\sqrt{1-t}}, \alpha_{1}(t)=\frac{1}{\sqrt[3]{t^{2}(1-t)}}, \alpha_{2}(t)=\frac{1}{\sqrt[3]{t(1-t)^{2}}}$ for all $t \in(0,1)$, $\beta_{1}(t, u, v)=(u+v)^{a}, \beta_{2}(t, u, v)=\ln (1+u+v)$ for all $t \in[0,1], u, v \geq 0$. For $c \in(0,1 / 2)$ fixed, the assumptions (H4), (H6), and (H8) are satisfied $\left(\beta_{i \infty}=0\right.$ for $i=1,2$ and $\left.\hat{f}_{\infty}=\infty\right)$.

Then by Theorem 3.6 , we deduce that there exists $\tilde{\lambda}_{*}>0$ such that for any $\lambda \geq \tilde{\lambda}_{*}$ our problem $\left(\mathrm{S}_{0}\right)-\left(\mathrm{BC}_{0}\right)$ (with $\lambda=\mu$ ) has at least one positive solution.

\section{Competing interests}

The authors declare that no competing interests exist.

\section{Authors' contributions}

The authors contributed equally to this paper. Both authors read and approved the final manuscript.

\section{Author details}

${ }^{1}$ Department of Mathematics, Baylor University, Waco, TX 76798-7328, USA. ²Department of Mathematics, Gh. Asachi

Technical University, lasi, 700506, Romania.

\section{Acknowledgements}

The authors thank the referees for their valuable comments and suggestions. The work of R Luca was supported by the CNCS grant PN-II-ID-PCE-2011-3-0557, Romania.

Received: 8 October 2015 Accepted: 2 March 2016 Published online: 08 March 2016

\section{References}

1. Das, S: Functional Fractional Calculus for System Identification and Controls. Springer, New York (2008)

2. Graef, JR, Kong, L, Kong, Q, Wang, M: Uniqueness of positive solutions of fractional boundary value problems with non-homogeneous integral boundary conditions. Fract. Calc. Appl. Anal. 15(3), 509-528 (2012)

3. Kilbas, AA, Srivastava, HM, Trujillo, JJ: Theory and Applications of Fractional Differential Equations. North-Holland Mathematics Studies, vol. 204. Elsevier, Amsterdam (2006)

4. Podlubny, I: Fractional Differential Equations. Academic Press, San Diego (1999)

5. Sabatier, J, Agrawal, OP, Machado, JAT (eds.): Advances in Fractional Calculus: Theoretical Developments and Applications in Physics and Engineering. Springer, Dordrecht (2007)

6. Samko, SG, Kilbas, AA, Marichev, Ol: Fractional Integrals and Derivatives: Theory and Applications. Gordon \& Breach, Yverdon (1993)

7. Henderson, J, Luca, R: Positive solutions for a system of fractional differential equations with coupled integral boundary conditions. Appl. Math. Comput. 249, 182-197 (2014)

8. Henderson, J, Luca, R, Tudorache, A: On a system of fractional differential equations with coupled integral boundary conditions. Fract. Calc. Appl. Anal. 18(2), 361-386 (2015)

9. Henderson, J, Luca, R: Positive solutions for a system of nonlocal fractional boundary value problems. Fract. Calc. Appl. Anal. 16(4), 985-1008 (2013)

10. Henderson, J, Luca, R: Existence and multiplicity of positive solutions for a system of fractional boundary value problems. Bound. Value Probl. 2014, 60 (2014)

11. Luca, R, Tudorache, A: Positive solutions to a system of semipositone fractional boundary value problems. Adv. Differ. Equ. 2014, 179 (2014)

12. Yuan, C, Jiang, D, O'Regan, D, Agarwal, RP: Multiple positive solutions to systems of nonlinear semipositone fractional differential equations with coupled boundary conditions. Electron. J. Qual. Theory Differ. Equ. 2012, 13 (2012)

13. Yuan, C: Multiple positive solutions for $(n-1,1)$-type semipositone conjugate boundary value problems of nonlinear fractional differential equations. Electron. J. Qual. Theory Differ. Equ. 2010, 36 (2010)

14. Agarwal, RP, Meehan, M, O'Regan, D: Fixed Point Theory and Applications. Cambridge University Press, Cambridge (2001)

15. Guo, D, Lakshmikantham, V: Nonlinear Problems in Abstract Cones. Academic Press, New York (1988) 